\title{
GA-stacking: Evolutionary stacked generalization
}

\author{
Agapito Ledezma*, Ricardo Aler, Araceli Sanchis and Daniel Borrajo \\ Computer Science Deparment, Universidad Carlos III de Madrid, Avda. de la Universidad, 30m 28911 \\ Leganés. Madrid, Spain
}

\begin{abstract}
Stacking is a widely used technique for combining classifier and improving prediction accuracy. Early research in Stacking showed that selecting the right classifiers their parameters and the meta-classifier was a critical issue. Most of the research on this topic hand picks the right combination of classifier and their parameters. Instead of starting from these initial strong assumptions, our approach uses genetic algorithms to search for good Stacking configurations Since this can lead to overfitting one of the goals of this paper is to empirically evaluate the overall efficien y of the approach. A second goal is to compare our approach with the current best Stacking building techniques. The results show that our approach find Stacking configuration that, in the worst case, perform as well as the best techniques, with the advantage of not having to manually set up the structure of the Stacking system.
\end{abstract}

Keywords: Ensembles of classifiers stacking, genetic algorithms, meta-classifier

\section{Introduction}

One of the currently favored lines of research in Machine Learning is the combination of classifier to improve classificatio accuracy [9]. This approach is known as ensembles of classifie $s$ in the supervised learning area. The main idea behind ensembles, is that they are often much more accurate than the individual classifier that make them up.

Typically, ensembles are constructed by generating several classifier with the same learning algorithm [10]. In order to generate different classifiers there are several methods that can be grouped in: sub-sampling the training examples (e.g. bagging [3] and boosting [18]); manipulating the input features [5]; manipulating the output target (e.g. ECOC [11]); and injecting randomness in the learning algorithm [26]. Once the classifier have been generated, they are combined, in most cases by voting or weighted voting.

Other research in the area uses different learning algorithms over a dataset to generate the ensemble members. One example of this approach is Stacking [39]. Stacking uses an algorithm to learn how to combine the outputs of a set of classifier that have been obtained by different learning algorithms. One of the problems of Stacking is how to obtain the right combination of base-level classifier and the meta-classifie, specially in relation to each specifi dataset. If the number of classifier and algorithms to be used is small, this problem can be solved by a simple method in reasonable time: exhaustive search. But, when the search space is large, it is difficul to fin the best Stacking configuration In a 


\section{A. Ledezma et al. / GA-stacking: Evolutionary stacked generalization}

previous paper [27], we presented an approach based on genetic algorithms (GAs) [21]. A somewhat related approach uses GAs for ensembles of neural networks, but only to determine the right number of ensemble members and in the context of homogeneous ensembles, not heterogeneous classifier generation algorithms like Stacking [40]. In an approach previous to Stacking, Wolpert [38] searched a space of generalizers with a particular version of an evolutionary algorithm. The search was restricted to the base generalizers. Also, English [15] used an evolutionary algorithm to explore the space of base classifier (recurrent neural networks only). This approach was tested in two time series problems.

There are also many variants of the basic Stacking algorithm. The methods that have reported the best results are Stacking with multi-response model trees [13] and Stacking with multi-response linear regression and a reduced set of attributes [32]. In this paper, we continue our line of thought, based on not imposing strong assumptions on the fina Stacking configuration and letting the GA fin an accurate one freely. In particular, we have extended our GA-Stacking approach by enlarging the search space of Stacking configurations taking into account not only the learning algorithms but also their parameters. This is easy to achieve in a genetic approach, by coding the parameters in the GA individual. We then compare results with two recent Stacking state-of-the-art approaches. We show that our approach is able to fin a Stacking configuratio that performs comparably well and in some case better than those state-of-art Stacking systems with the advantage of not having to set manually an appropriate selection of algorithms and parameters.

This paper is organized as follows: Section 2 gives some background on Stacking and recent Stacking variants. Section 3 introduces the Stacking configuratio approach, GA-stacking. Section 4 describes the experimental evaluation of the GA-Stacking viability. Section 5 and Section 6 show the results of the performed experiments carried out. Section 7 draws some conclusions. Finally, Appendices A and B show the parameters of the learning algorithms used by GA-Stacking.

\section{Stacking Approaches}

In this Section, we firs describe the Stacking framework and then we present a brief description of some recent work on stacking.

\subsection{Stacked Generalization}

Stacking is the abbreviation that refers to Stacked Generalization [39]. The main idea of Stacking is to combine classifier from different learners such as decision trees, instance-based learners, etc. Since each one uses a different knowledge representation and different learning biases, the hypothesis space will be explored differently, and different classifier will be obtained. Thus, it is expected that their errors will not be perfectly correlated, and that the combination of classifier will perform better than the base classifiers

Once the classifier have been generated, they must be combined. Stacking uses the concept of the meta learner. The meta learner (or level-1 model), tries to acquire, using a learning algorithm, how the outputs of the base classifier (or level-0 models) should be combined to obtain the fina classification This is achieved by a cross-validation-like process, as described in [39].

More formally, given a data set $S$, Stacking firs generates a subset of training sets $S_{1}, \ldots, S_{T}$ and then follows something similar to a cross-validation process: it leaves one of the subsets out (e.g. $S_{j}$ ) to be used later. The remaining instances $S^{(-j)}=S-S_{j}$ are used to generate the level-0 classifier by applying $K$ different learning algorithms, $k=1, \ldots, K$, to obtain $K$ classifiers After the level-0 models 
have been generated, the $S_{j}$ set is used to train the meta learner (level-1 classifier) Level-1 training data is created from the predictions of the level- 0 models over the instances in $S_{j}$, that were left out for this purpose. Level-1 data has $K$ attributes, whose values are the predictions of each one of the $K$ level-0 classifier for every instance in $S_{j}$. Therefore, a level-1 training example is made of $K$ attributes (the $K$ predictions) and the target class, which is the right class for every instance in $S_{j}$. Once the level-1 data has been built from all instances in $S_{j}$, any learning algorithm can be used to generate the level-1 model. To complete the process, the level- 0 models are re-generated from the whole data set $S$ (this way, it is expected that classifier will be slightly more accurate). To classify a new instance, the level-0 models produce a vector of predictions that is the input to the level-1 model, which in turn predicts the class.

\subsection{Related Work}

Recent work on Stacking addresses the Stacking configuratio problem: what algorithm and features are to be used in the meta-level. We present here a brief review of these work.

A necessary condition to create a good ensemble of classifier is that base-level classifier error predictions are uncorrelated [20]. In [29] a variant of stacking is proposed that uses correspondence analysis in order to detect correlations between the base-level classifiers Once dependencies have been removed from the original meta-level space, a nearest neighbor method (meta-level algorithm) is then applied over the resulting features space. This approach is called SCANN.

In [34], they use probability distributions for the outputs from level-0 models instead of a simple class prediction as meta-level attributes. By using probability distributions as meta-level data, the authors argue that both, prediction and confidenc of the base-level classifiers are used. In order to use this type of meta-level data, the authors proposed to use the multi-response linear regression technique (MLR) as the meta-level algorithm.

Another variant of Stacking [33] creates a meta-level classifie for each level-0 classifie . The learning task for each level-1 classifie is to predict whether the level-0 classifie prediction will be correct. The meta-level data is composed of base-level attributes and the class values are correct or incorrect. The predictions of those classifier are combined by summing up the predicted probability distribution.

In [36] a new variant of Stacking is described that uses another learning method in the meta-level. This method, called meta decision trees (MDTs), replaces class-value predictions in its leaf nodes by the base level-classifiers The meta-level data is composed of properties of the probability distributions that reflec the confidenc of the base-level classifiers like entropy and maximum probability, rather than the distributions themselves. Based on these properties, small MDT's are generated.

Based on Stacking with MLR (SMLR), [32] proposed to reduce the number of meta-level attributes independently of the number of classes, in order to overcome a weakness of SMLR in domains with more than two classes. This method is called StackingC (STC).

Džeroski and Ženko [14] proposed two new variants of stacking. First, based on Stacking with MLR, they propose to extend the set of meta-level features augmented with the probability distribution multiplied by the maximum probability and the entropies of the probability distributions. On the other hand, they propose another extension of SMLR in which they use model tree induction instead of linear regression as the meta-level algorithm. This method is called Stacking with multi-response model trees (SMRMT).

Comparing Stacking approaches overall, SCANN, MDTs, SMLR and SelectBest (selecting the best classifie with cross-validation) seem to perform at about the same level [14]. Moreover, they concluded that Stacking with multi-response model trees (SMRMT) performs better than existing Stacking approaches, including STC, and selecting the best classifie from the ensemble by cross-validation. 
One of the main conclusions of all this previous work is that there are many contradictory results and there is no consensus on which combination of classifier is the best one. The main differences of previous work with respect to our approach are that we do not select "a priori":

- which meta-classifie to use,

- the parameters of the meta-classifie,

- the number of base classifiers

- which of the available base classifier to use, nor

- the parameters of these base classifier

The main advantage of our approach is its fl xibility and its non "a-priori" commitments. The system is very extensible. It can benefi from new classifiers since they can be easily incorporated into the pool of available classifier together with their parameters, and coded in the GA-Stacking chromosome. Another advantage of our approach is that it is dataset dependent, so we also adapt to dataset biases and features, while the rest of approaches use the same configuratio for all datasets.

\section{GA-Stacking}

Since an ensemble of classifier generated from Stacking is composed by a group of models created from different learning algorithms, the following question arises: what algorithm should be used to generate the level-0 and the level-1 models?. Wolpert [39] originally indicates that many aspects on the parameters of Stacking, including the algorithms that generate the classifiers can be considered as black art. At first any learning algorithm can be used to generate the classifier for both levels. As we described in the previous section, there have been some work focused on providing answers to these questions. For example, Ting and Witten [35] showed that a linear model is useful to generate the level-1 classifie when outputs of level-0 classifier are probabilistic, Seewald [32] proposes a variation in the level-1 attributes space, Džeroski and Ženko [14] propose a regression tree as the meta-classifie instead of the linear model proposed by Ting and Witten.

Based on the idea of using probability class distributions as meta-level data proposed by Ting and Witten [35], we propose a new approach based on genetic algorithms which searches for the optimal configuratio of Stacking parameters in a given problem. In Section 3.1 we describe the general framework of our approach. In Section 3.2 we detail the solution encoding for our GA-based system. Finally, in Section 3.3 we show the evaluation process of solutions found by the GA.

\subsection{General Framework}

GA-Stacking is the acronym of Genetic Algorithms for Stacking. GA-Stacking provides answers to the previous questions: which and how many learning algorithms are necessary to generate base-level classifiers and, what algorithm should be used to generate the meta-level classifier? The proposed solution consists on considering them as an optimization problem, which can be solved by applying Genetic Algorithms. Figure 1 shows the proposed general framework.

The application of GAs to an optimization problem requires, mainly, the study of two aspects: the encoding of the candidate solutions and the definitio of the fitnes function. The process of codificatio of the solutions takes place before (Fig. 1, Phase I) the execution of the GA. With respect to the fitnes evaluation, it is an iterative process that is carried out in each generation of the GA (Fig. 1, Phase II) on all the individuals $(p)$ of the population $(P)$. The encoding-solutions phase is detailed in Section 3.2. The fitnes evaluation is detailed in Section 3.3. 


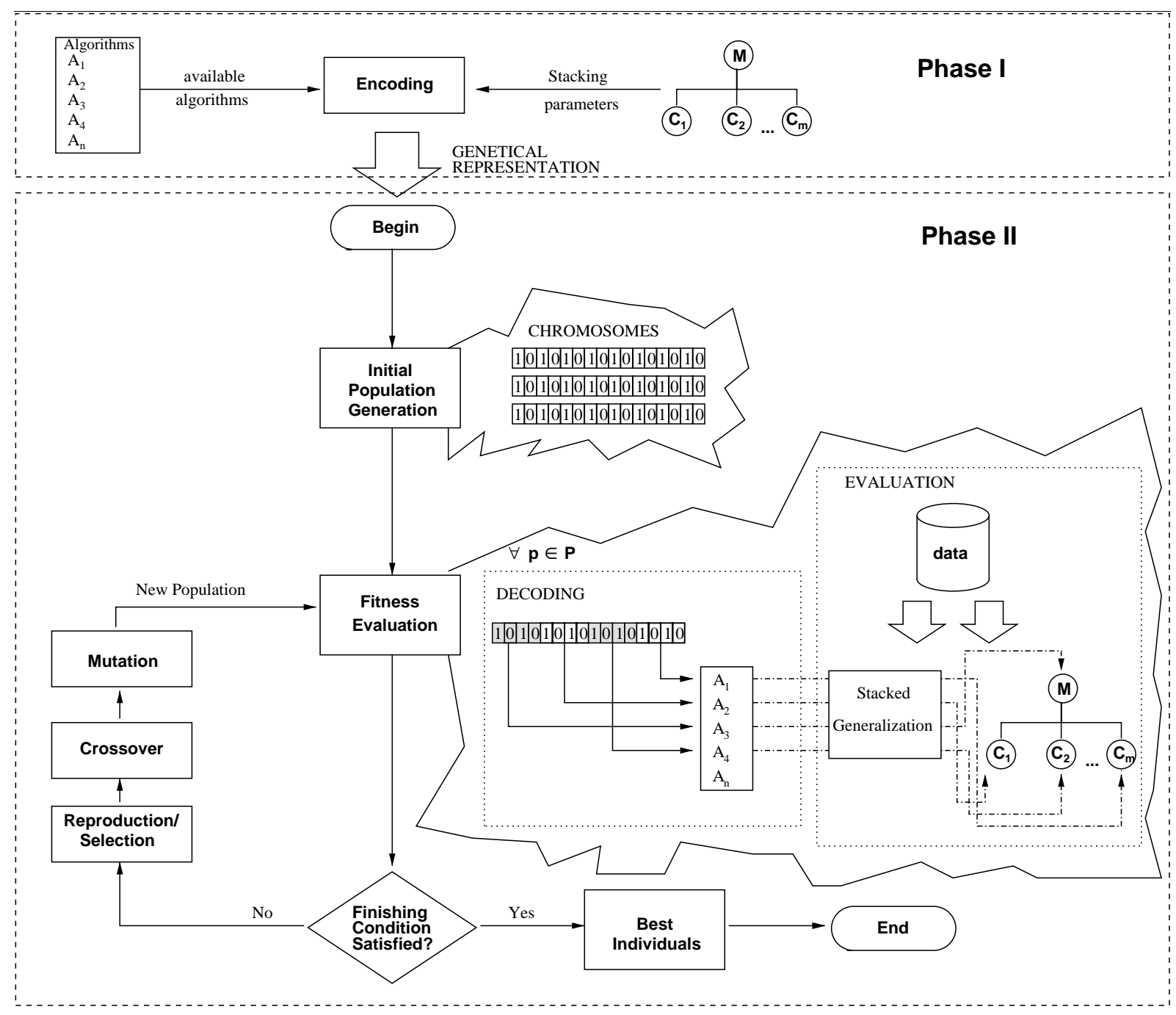

Fig. 1. Proposed framework GA-Stacking.

\subsection{Encoding of Candidate Solutions}

There are different ways to represent the solutions of a problem for a GA (e.g. binary, decimal, hexadecimal, etc. codifications) In order to represent the candidate solutions or individuals in GA-Stacking, we decided to use a binary representation, since it allows the use of canonical GAs. The canonical GA is the original form proposed by Holland $[21,22]$ and has a stronger mathematical foundation $[19,30]$ ) which relies on binary codifications

The second decision consists on selecting the size of the chromosomes, which is given by two factors in our case:

- the number of algorithms that can be selected in order to generate the base-level and meta classifier $(m)$.

- the maximum number of base-level classifier in the ensemble $(n)$. 


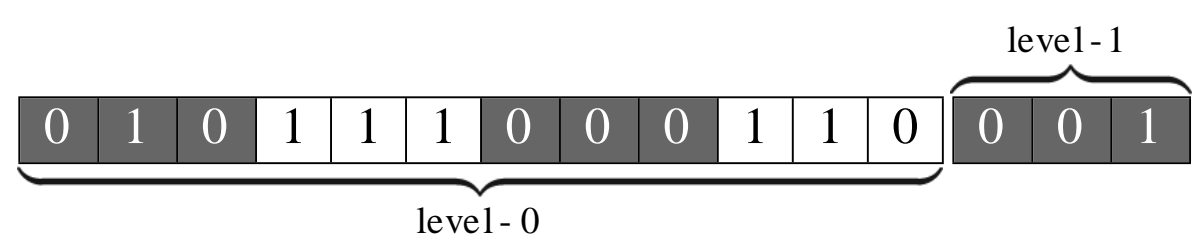

Fig. 2. A binary string representing a combination of four base-level classifiers and one meta-level classifie .

If we consider only the classifie name in the coding process, the gene size depends on the available learning algorithms. For example, if there are seven learning algorithms available to generate the members of the ensemble, we can use three bits to represent a gene. In this case, the gene can represent the use of any of the seven algorithms, and the option of using none. Figure 2 shows an individual in which the firs four genes of the chromosome represent the four learning algorithms used to generate the base-level classifier and, the last gene represents the algorithm used to create the meta-classifie .

In addition to the identifie of an algorithm, we also want to represent the algorithm's parameters (like the number of hidden neurons in a neural network). In that case, more than one gene will be needed per algorithm. If there are $n$ base classifier and $G$ is the number of genes per classifie (including parameters), the total number of genes per individual, $T_{c}$, is given by

$$
T_{c}=G \times(n+1)
$$

Concerning the length of the chromosome in bits, it derives from the number and size of genes used to represent the available algorithms able. In other words the length of the chromosome in bits is given by

$$
T_{b}=(n+1) \times \sum_{i=1}^{G} x_{i}
$$

where $x_{i}$ represents the number of bits used for coding the gene $i$. For example, the individual shown in Fig. 2 has $G=1, n=3$ and $x_{i}=3$.

\subsection{Fitness Evaluation}

The fitnes evaluation process is carried out in two phases: the decoding and construction phase; and the fitnes calculation phase. In the firs phase, decoding and ensemble construction (Fig. 3[a]), we generate the ensemble of classifier using the algorithms represented in the chromosome and the training dataset. The firs step is to extract the identifie and the parameters for each algorithm from the chromosome. After that, we use a subset of available domain data (training data) to generate the ensemble.

In the second phase (Fig. 3 [b]), the fitnes calculation, we use a different dataset (validation data) in order to estimate the fitnes value of each individual. The fitnes is the accuracy on the validation dataset.

\subsection{Setting the GA parameters}

In addition to the codificatio type and the number of genes in the chromosome, GAs have other parameters. Structural parameters like the population size, and execution parameters like mutation and elite rate, have to be set. Nevertheless, in this work, we have used the most frequently values for this parameters [19] yielding good results. 


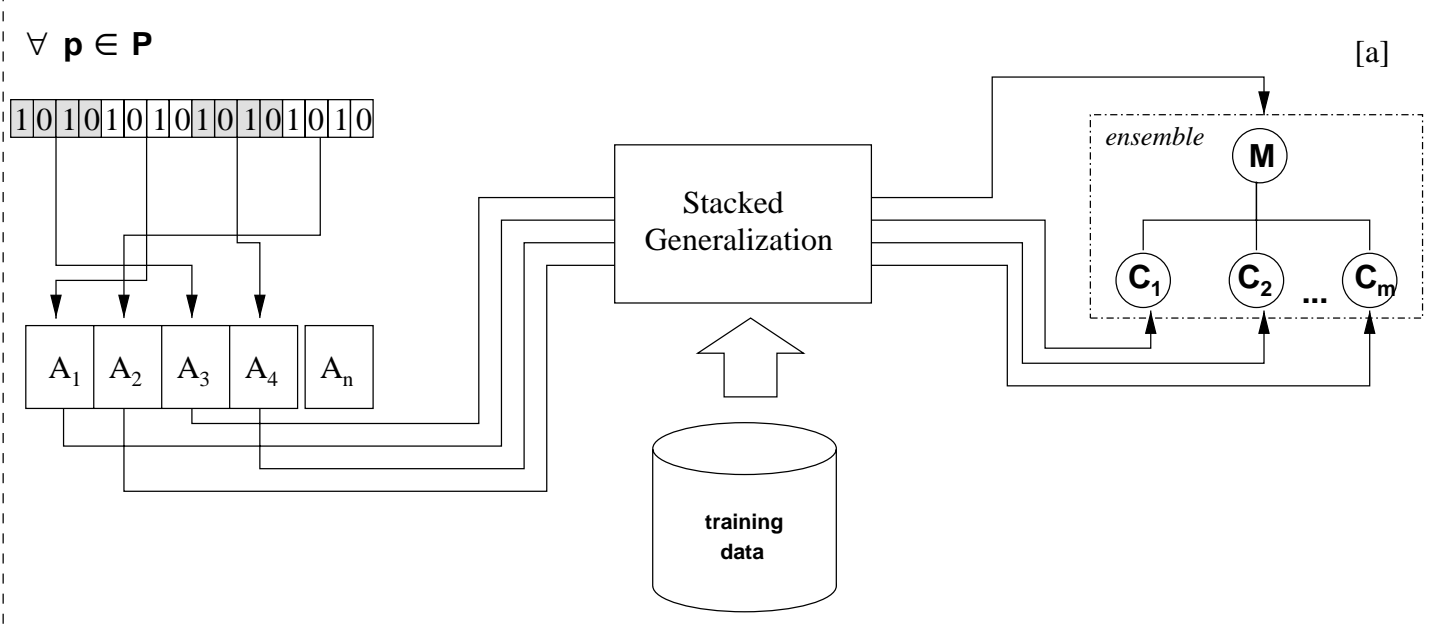

Decoding and Ensemble Generation

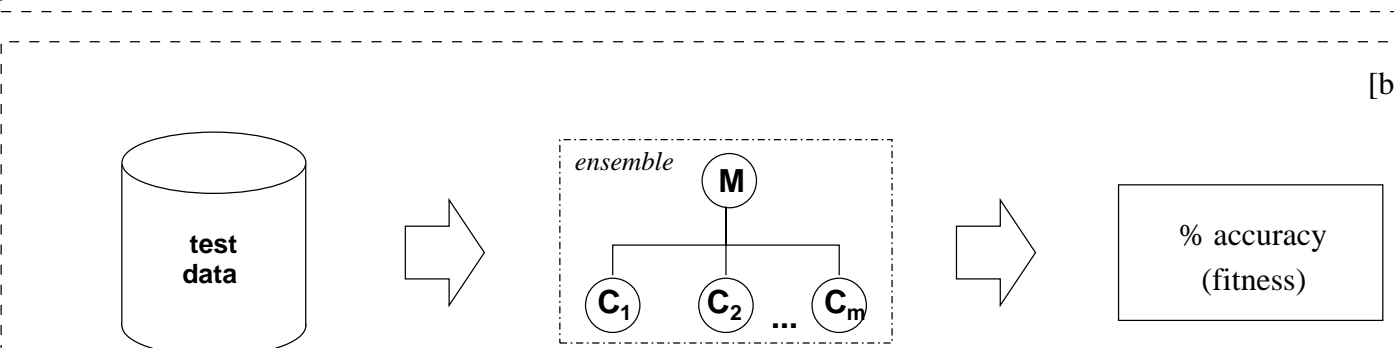

Fitness Evaluation

Fig. 3. Fitness computation in GA-Stacking.

\section{GA-Stacking viability evaluation}

In order to evaluate our approach we carried out two simple preliminary experiments to show the initial validity of GA-Stacking. In order to carry out this evaluation we used several domains from the UCI repository [2]. At a firs stage we obtained promising results, but there were signs of overfitting In order to avoid overfitting we carried out another set of experiments with a different fitnes evaluation process. In all these experiments, we used the learning algorithms implemented in the WEKA suite [37] (version 3.1.7). WEKA includes all algorithms used for experimentation including the ensemble generation algorithms (i.e. Bagging, Boosting and Stacking).

The implementation of GA-Stacking has two parts: the learning algorithms for which we used WEKA; and the GA, for which we used the GAJIT library (Genetic Algorithm Java Implementation Toolkit) [16].

\subsection{Preliminary results}

In this Section we show the results of GA-Stacking over two domains from the UCI repository: ionosphere and dermatology, given that they have been widely used on other comparisons related to ensembles of classifiers Table 1 shows the values of the GA parameters used in this experiment. 
Table 1

GA parameters

\begin{tabular}{lr}
\hline Parameter & Value \\
\hline Population & 10 \\
Generations & 10 \\
Elite rate & 0.10 \\
Cull rate & 0.40 \\
Mutation rate & 0.067 \\
\hline
\end{tabular}

Given that we are only studying the viability of the system and that the time to run the experiments is large, we have only considered here a small population size and a small number of generations. Regarding to the elite, cull and mutation rates, we use the defaults values of the GAJIT library.

We have selected seven algorithms in order to generate the ensemble of classifiers

- C4.5 [31]. It generates decision trees - (C4.5).

- A probabilistic Naive Bayes classifie [24] (NB).

- A simple Naive Bayes classifie where numerical attributes are modeled by a normal distribution [12] (NBS).

- IB $k$ [1]. This is Aha's instance-based learning algorithm - (IB k). Default value of $k$ is 1 .

- PART [17]. It creates decision lists from partially pruned decision trees, generated using the C4.5 heuristic - (PART).

- Decision Table [25]. It is a simple classifie that uses the majority class. - (DT).

- Decision Stump [23]. It generates one-level decision trees - (Ds).

On the other hand, we have used two algorithms to generate the ensemble in order to compare the results of GA-Stacking with other ensembles of classifiers The algorithms are:

- Bagging: algorithm for creating homogeneous ensembles of classifier based on subsampling the training set. In this experiment we used C4.5 as the base classifie .

- Boosting: algorithm for creating homogeneous ensembles of classifier based on weighting training instances. We used AdaBoostM1 with C4.5 as the base classifie.

In order to evaluate the individual found by GA-Stacking, we performed the following:

- Each dataset was randomly divided into two parts, $A$ and $B$

- Part $A$ (around $85 \%$ of available domain instances) was used as the training set, and as the fitnes evaluation set.

- In order to compute the fitnes of each individual in the population, we generate an ensemble of classifier using Stacking. The Stacking parameters (learning algorithms) are codifie in the chromosome. Once the ensemble of classifier has been generated, we use the accuracy of the ensemble over the training set (set $A$ ) as the fitnes value.

- The dataset $B$ was used as test set. Since these experiments were preliminary, the fitnes evaluation process has not been carried out as we described in Section 3.3, where we proposed to split the training data in two sets in order to carry out a cross-validation.

Results are shown in Table 2. Columns two and four show the accuracy on the training data of the ensemble of classifier in the ionosphere and dermatology domains. Columns three and fi e show the results over the test datasets.

The top rows of the table show the results of using the available individual learning algorithms. In the center rows of the table, we show the results of Bagging and Boosting and the best individual found 
A. Ledezma et al. / GA-stacking: Evolutionary stacked generalization

Table 2

GA-Stacking evaluation: preliminary results

\begin{tabular}{lrrrr}
\hline \multirow{2}{*}{ Algorithm } & \multicolumn{2}{c}{ Ionosphere } & \multicolumn{2}{c}{ Dermatology } \\
\cline { 2 - 5 } & Training & Test & Training & Test \\
\hline Single & & & & \\
C4.5 & 98.42 & 82.86 & 96.67 & 92.42 \\
Naive Bayes & 85.13 & 82.86 & 98.67 & $\mathbf{9 6 . 9 7}$ \\
Naive Bayes Simple & 85.13 & 82.86 & 98.67 & $\mathbf{9 6 . 9 7}$ \\
PART & 98.73 & 88.57 & 96.67 & 93.94 \\
IB1 & 100.00 & 82.86 & 100.00 & 92.42 \\
Decision Stump & 84.18 & 80.00 & 51.33 & 45.45 \\
Decision Table & 94.94 & 88.57 & 96.67 & 87.88 \\
Ensembles & & & & \\
Bagging with C4.5 & 97.78 & 85.71 & 97.00 & 93.94 \\
Boosting with C4.5 & 100.00 & $\mathbf{9 1 . 4 3}$ & 100.00 & $\mathbf{9 6 . 9 7}$ \\
Final GA-Stacking & 100.00 & 85.71 & 100.00 & 95.45 \\
Intermmediate GA-Stacking & 97.78 & 94.29 & 98.67 & 98.48 \\
\hline
\end{tabular}

by GA-Stacking after all generations. The individual found by GA-Stacking obtains a $100 \%$ of accuracy over the training set in both domains. However, the results over the test sets $(85.71 \%$ for ionosphere and $95.45 \%$ for dermatology) show a potential overfitting In the ionosphere domain the result obtained by the fina GA-Stacking individual is worse than PART, Decision Table and Boosting. In the dermatology domain the results of the fina GA-Stacking individual is surpassed by the classifier generated from Naive Bayes and Boosting, both obtaining the best results in this domain (96.97\%).

However, if we analyze the evolution of the fitnes value of intermediate individuals, we can see that in previous generations GA-Stacking found individuals that obtained better results than any single or ensemble classifie over the test dataset in both domains (last row of Table 2). This reinforces the idea that GA-Stacking overfit to the training data.

Figure 4 shows the fitnes evolution in the dermatology domain. In the third generation, there is an individual that has the maximum fitnes value (100\% of accuracy), but the results over the test set get worse from this generation onwards. Figure 4 also shows the average accuracy of the best three individuals of each generation in training/fitnes and the results over the test set. The fitnes average reaches $100 \%$ of success in the sixth generation, but the accuracy in test had already been decreasing.

\subsection{Avoiding Overfittin}

As we just saw, the Stacking configuration found by GA-Stacking overfitted One of the reasons for this behavior, might be that the fitnes value is obtained using the same instances employed to generate the ensemble of classifier by means of Stacking. In order to avoid overfitting we designed a new set of experiments where the fitnes value is computed from a new dataset (validation dataset). In other words, the training set was split randomly into two datasets. $80 \%$ of the original training instances are used to generate the ensemble of classifier from the configuratio found by GA-Stacking, and the rest of instances - the validation dataset - are used to estimate the fitnes value. This scheme has the disadvantage that the ensemble of classifier is generated from fewer instances than previous experiments.

In the following experiments we extended the number of domains in order to evaluate GA-Stacking. We used six domains from the UCI repository. These domains are define in Table 3.

We used the same GA parameters as in the previous set of experiments (see Table 1). A fi e-fold cross-validation was performed. For experimental reasons we execute the GA only one time for each fold. The classificatio accuracy of each classifier/ensemble $C$, over a given domain is the average 


\section{A. Ledezma et al. / GA-stacking: Evolutionary stacked generalization}

Table 3

Domains used for GA-Stacking evaluation

\begin{tabular}{lclcc}
\hline Domain & Attributes & Attributes Type & Instances & Classes \\
\hline dermatology & 34 & numeric-nominal & 366 & 6 \\
dna-splice & 60 & nominal & 3190 & 3 \\
heart & 13 & numeric-nominal & 303 & 2 \\
ionosphere & 34 & numeric & 351 & 2 \\
musk & 166 & numeric & 476 & 2 \\
sonar & 60 & numeric & 208 & 2 \\
\hline
\end{tabular}

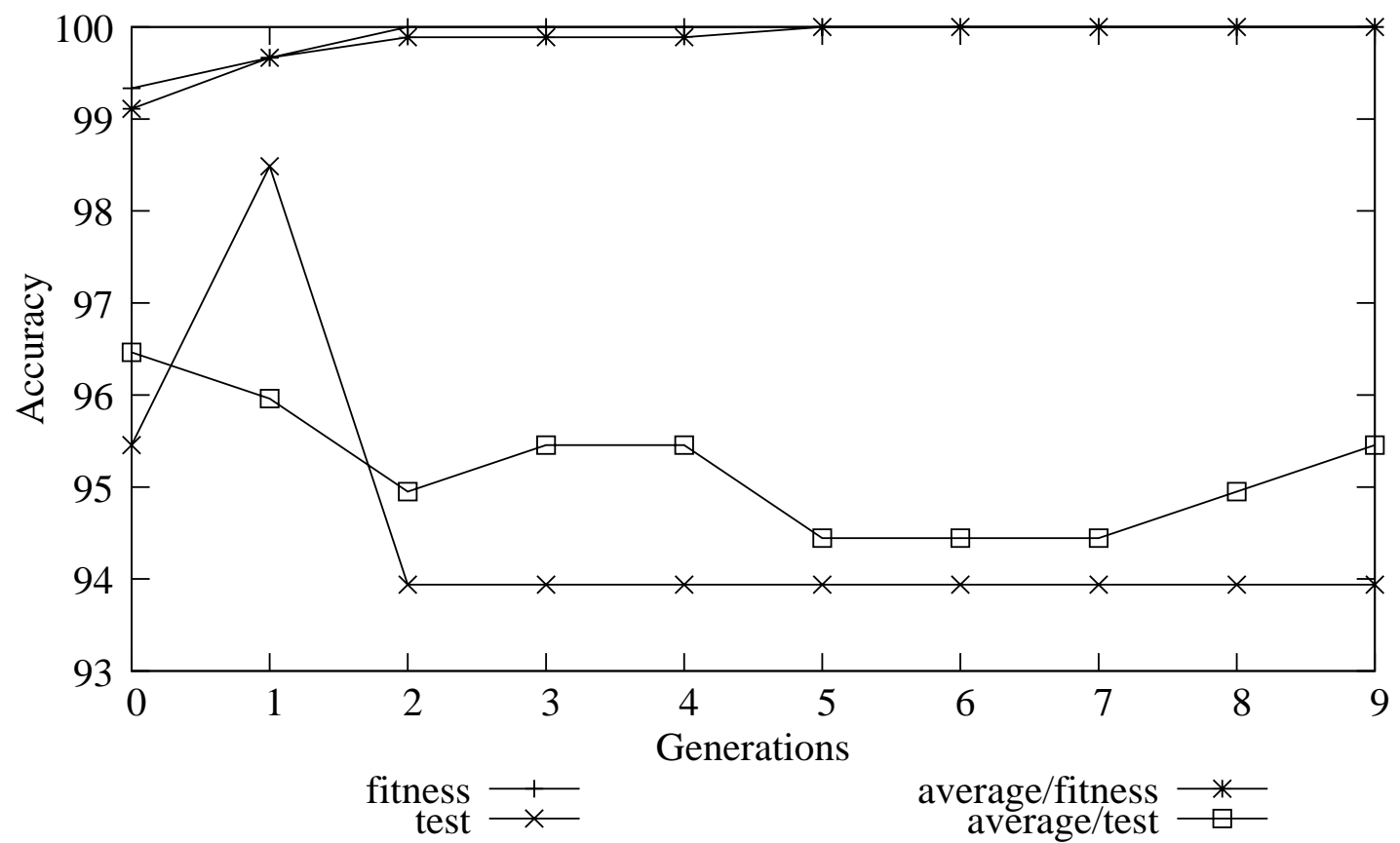

Fig. 4. Fitness evolution in the dermatology domain (best individual and the average of the three best individuals in each generation).

of the cross-validation $\left(A_{C}\right)$. In order to compare two learning algorithms, we have used the relative improvement and the paired t-test as it is described next.

In order to obtain the relative improvement of a given classifier/ensembl $\left(C_{1}\right)$ over another classifi er/ensemble $\left(C_{2}\right)$, we use $1-\operatorname{error}\left(C_{1}\right) / \operatorname{error}\left(C_{2}\right)$. The classifier/ensembl error is given by $1-A_{C}$. The average relative improvement (ARI) over all domains is computed using the geometric mean of the error reduction in the single domains as in [14]:

$$
1-\text { geometricMean }\left(\frac{\operatorname{error}\left(C_{1}\right)}{\operatorname{error}\left(C_{2}\right)}\right)
$$

The statistical significanc is estimated using a paired t-test with $\alpha=0.05:+/-$ in the results tables indicate that $C_{1}$ is better/worse than $C_{2}$. Table 4 shows the results of the individual classifier in all domains, and Table 5 shows the results of the ensemble methods, including GA-Stacking (best results are in bold). 
Table 4

Accuracy rate of individual classifier

\begin{tabular}{lcccccc}
\hline Domain & C4.5 & PART & NB & IB1 & DT & DS \\
\hline dermatology & 94.33 & 94.59 & 97.03 & 94.59 & 87.83 & 50.40 \\
dna-splice & 94.12 & 92.11 & 95.63 & 75.62 & 92.49 & 62.42 \\
heart & 74.00 & 82.00 & $\mathbf{8 3 . 0 0}$ & 78.00 & 76.33 & 70.67 \\
ionosphere & 90.14 & 89.30 & 82.82 & 87.61 & 89.30 & 82.82 \\
musk & 83.33 & 85.21 & 74.38 & 86.46 & 82.08 & 71.46 \\
sonar & 72.38 & 73.81 & 67.62 & 79.52 & 71.90 & 73.93 \\
\hline
\end{tabular}

Table 5

Accuracy rate of ensemble methods

\begin{tabular}{lccc}
\hline Domain & Bagging & Boosting & GA-Stacking \\
\hline dermatology & 94.59 & 97.03 & $\mathbf{9 7 . 3 0}$ \\
dna-splice & 94.56 & 94.43 & $\mathbf{9 5 . 7 2}$ \\
heart & 76.33 & 79.67 & 80.67 \\
ionosphere & $\mathbf{9 2 . 1 1}$ & 91.83 & 90.42 \\
musk & 87.29 & $\mathbf{8 8 . 9 6}$ & 83.96 \\
sonar & 80.00 & 79.05 & $\mathbf{8 0 . 4 8}$ \\
\hline
\end{tabular}

Table 6

Accuracy relative improvement (in \%) of the hypotheses found by GA-Stacking comparing them with the different single classifier and homogeneous ensemble methods (Bagging and Boosting) and the statistical significanc $(+/-$ is better/worse, ' ' not significant

\begin{tabular}{lrrrrrrrr}
\hline Domain & C4.5 & PART & \multicolumn{1}{c}{ NB } & IB1 & DT & DS & Bagging & Boosting \\
\hline heart & 25.64. & -7.41. & -13.73. & 12.12. & 18.31. & $34.09+$ & $18.31+$ & 4.92. \\
sonar & 29.31. & 25.45. & $39.71+$ & 4.65. & 30.51. & 26.79. & 2.38. & 6.82. \\
musk & 3.75. & -8.45. & $37.40+$ & -18.46. & 10.47. & $43.80+$ & -26.23. & -45.28. \\
ionosphere & 5.77. & 7.92. & $42.64+$ & 20.48. & 7.92. & $42.64+$ & -24.96. & -20.65. \\
dermatology & 52.39. & $50.01+$ & 9.12. & $50.01+$ & $77.78+$ & $94.54+$ & 50.01. & 9.11. \\
DNA splice & $27.27+$ & $45.82+$ & 2.16. & $82.45+$ & $43.10+$ & $88.62+$ & $21.39+$ & $23.16+$ \\
Average & 25.94 & 22.51 & 22.37 & 35.92 & 37.58 & 68.55 & 11.08 & -1.36 \\
win/lose & $1+/ 0-$ & $2+/ 0-$ & $3+/ 0-$ & $2+/ 0-$ & $2+/ 0-$ & $5+/ 0-$ & $2+/ 0-$ & $1+/ 0-$ \\
\hline
\end{tabular}

With the exception of the heart domain, the ensemble methods obtain better results than any single algorithm. Also, the ensembles of classifier generated from the Stacking configuratio found by $G A$ Stacking obtain better results in three out of the six domains, showing that it is a viable approach.

Table 6 shows a comparison among the ensemble of classifier found by GA-Stacking, the individual learning algorithms and Bagging and Boosting. The solutions generated from GA-Stacking improve, on average, over all the individual classifier and Bagging, but not Boosting. Nevertheless, if we analyze the statistical significanc of the results, the solutions of GA-Stacking are not significantl worse than any of the single classifiers or any of the methods for ensemble generation in any domain. On the other hand, GA-Stacking is better, significantl, than any of the single classifier or the techniques for ensemble generation in at least one domain, as it can be seen in the last row of Table 6.

Regarding the evolution of fitness Fig. 5 shows a comparison of the use of the same dataset both to generate the ensemble of classifier and to compute the fitnes value (a), and the use of a different dataset in order to evaluate the fitnes (b). As we can see, the accuracy rate over the test set increases if training and validation sets are used to generate the ensemble of classifiers Also, if we analyze the evolution of fitness the use of two datasets avoids, to a certain extent, the overfitting

Figures 6 and 7 show the evolution of fitnes together with the training and test accuracies in the domains used in these experiments. $100 \%$ accuracy is not reached in any training or fitnes dataset. 


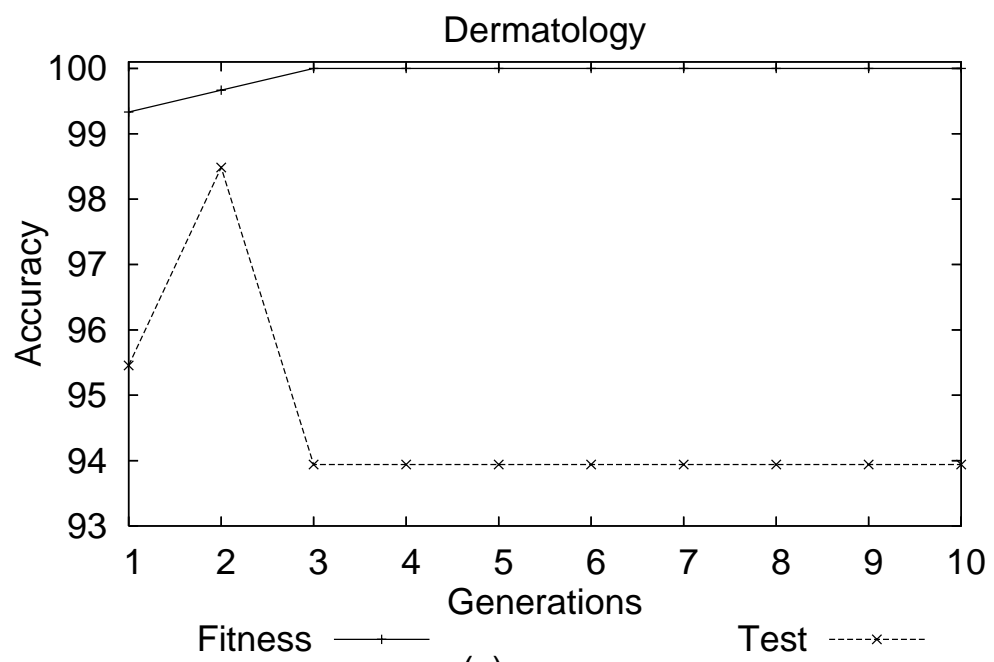

(a)

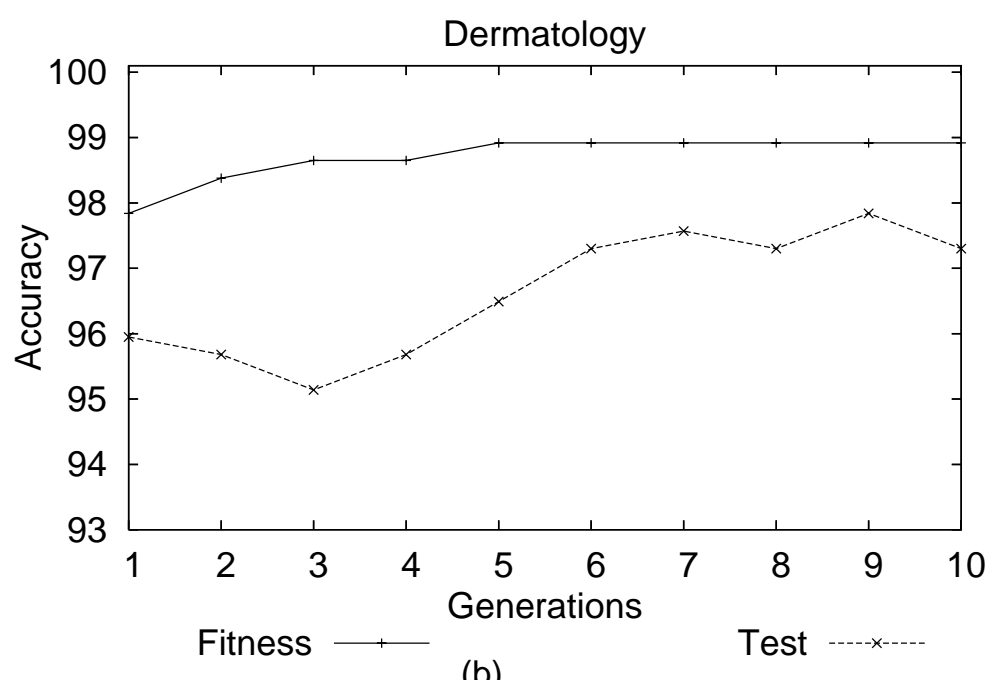

(b)

Fig. 5. Comparison of the evolution of fitnes using the same dataset for training and fitnes calculation $(a)$ or different datasets (b) in the Dermatology domain.

Thus, overfittin seems to be prevented.

\section{Evolving the GA-Stacking parameters}

Once the viability of using GAs to obtain good Stacking configuration has been established, we intend to evolve, not only better GA-Stacking configurations but also the parameters of the base and meta-classifier present in that configuration This would include, for instance, the number of hidden neurons for ANN classifiers In Sections 5.1, 5.2 and 5.3 we detail all parameters and classifier that GA-Stacking is able to evolve. In Section 5.4 we show the experimental setup and in Section 5.5 we detail the results obtained when comparing different configurations 
A. Ledezma et al. / GA-stacking: Evolutionary stacked generalization
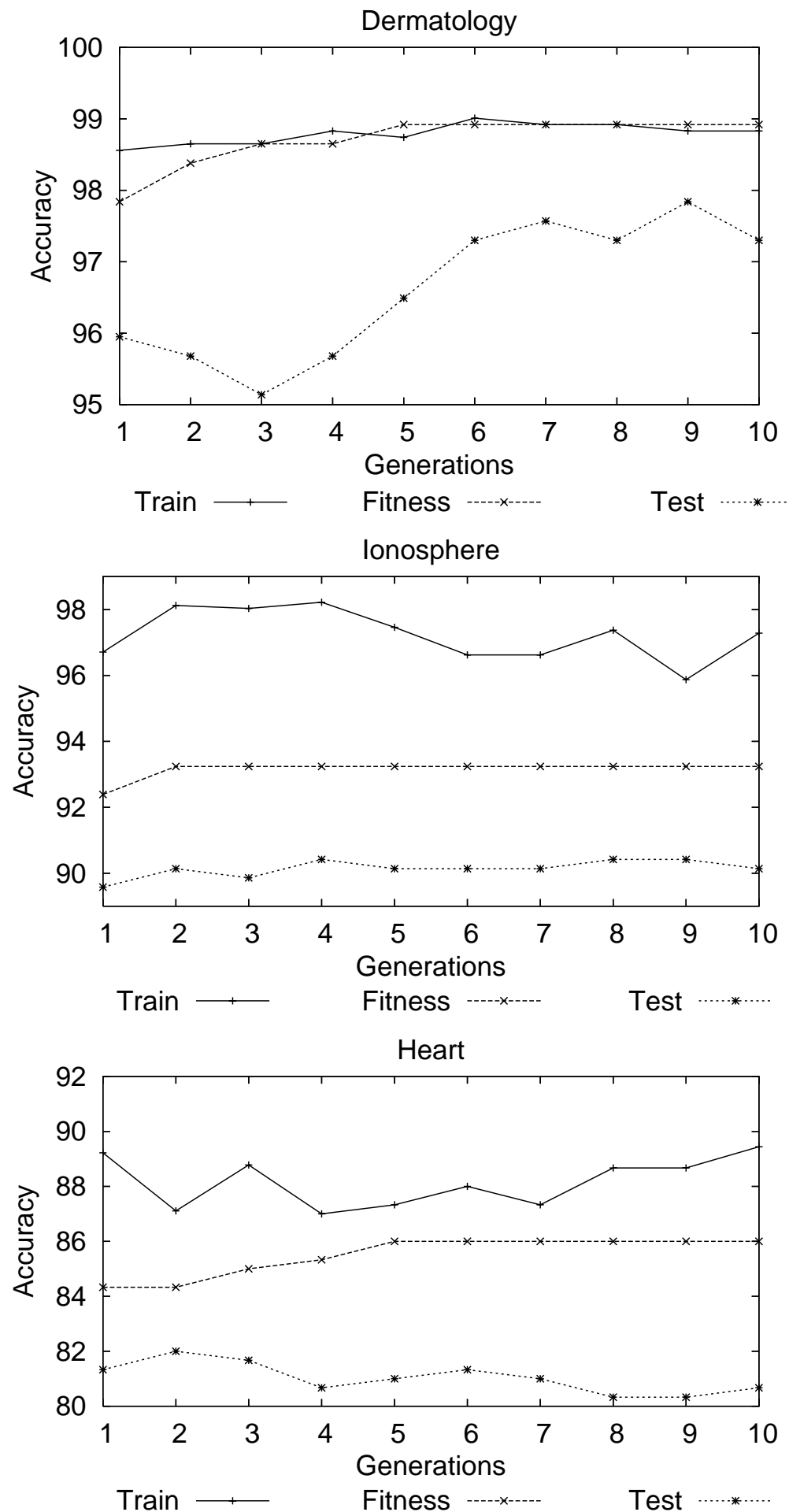

Fig. 6. Fitness evolution compared to the training and test accuracies in dermatology, ionosphere and heart domains. 

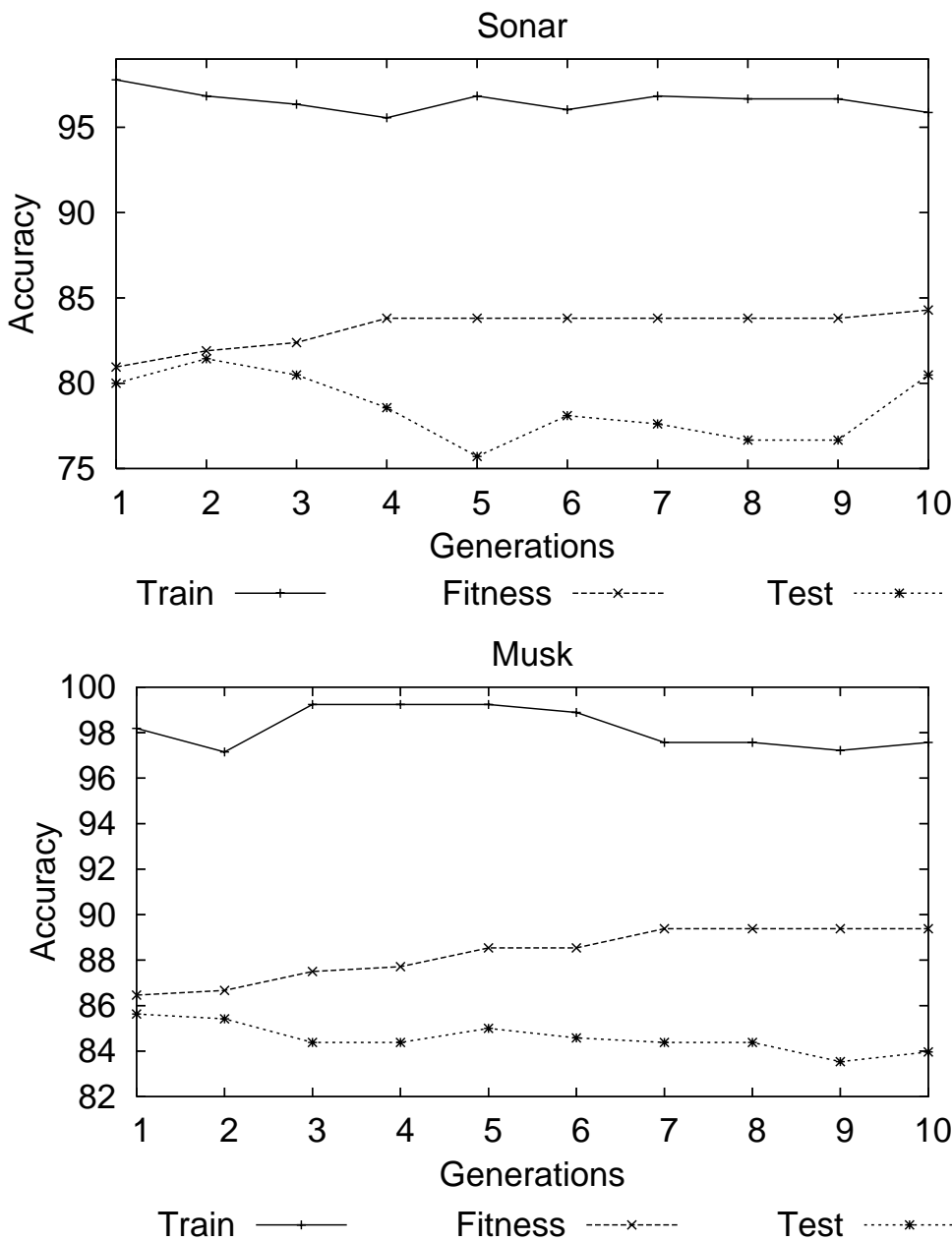

DNA Splice

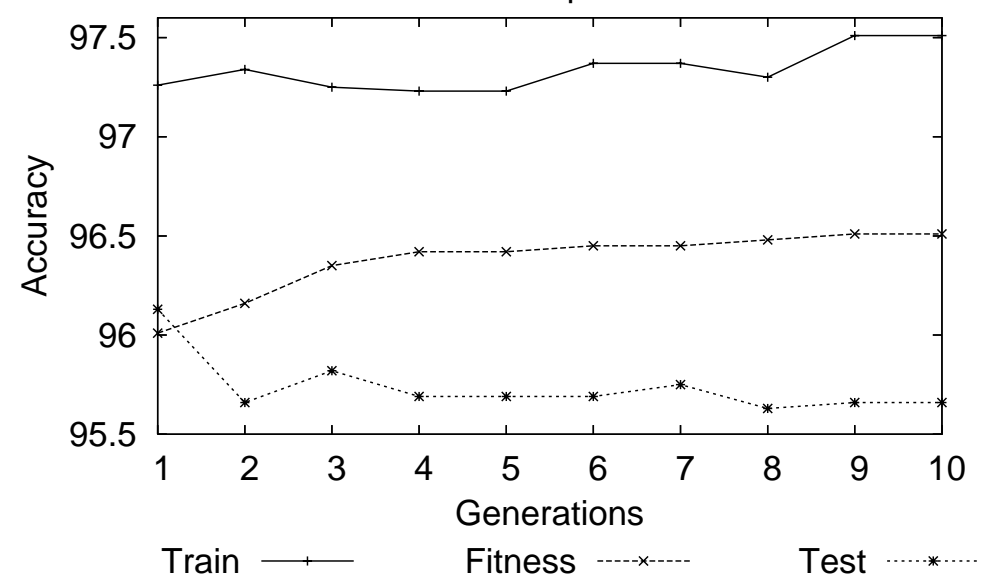

Fig. 7. Fitness evolution compared to the training and test accuracies in the sonar, musk and DNA domains. 


\subsection{Learning Algorithms}

In order to extend the configuration space in which the GA carries out the search of the good Stacking parameters configurations we extended the number of possible learning algorithms that can be used to generate the ensemble members. These algorithms can be used to generate both the base classifier and the meta-classifie. In addition to the algorithms used in the firs experiments, C4.5, Naive Bayes, IB $k$, PART, Decision Stump and Decision we have incorporated the following algorithms:

- Random Forest [4]. This algorithm constructs a Random Forest by combining many unpruned decision trees, RF.

- Random Tree [37]. This algorithm constructs a tree considering $K$ random attributes at each node. It does not carry out any pruning, RT.

- MLR [34]. A multi-response lineal regression, MLR.

- MRMT [13]. A multi-response model tree, MRMT.

- K* [6]. An instance-based algorithm that uses a distance based on the entropy, $\mathrm{K}^{*}$.

- VFI [8]. An algorithm that generates a classifie that classifie an instance based on features intervals, VFI.

- Conjunctive Rule. This algorithms generates a simple conjunctive rules classifie, CR.

- JRip [7]. An algorithm that generates propositional rules, JRIP.

- Nnge [28]. It is a nearest neighbor algorithm that uses non-nested generalized exemplars, NNGE.

- HyperPipes [37]. It generates a classifie that constructs a HyperPipe for each category, which contains all the points of that category, HP.

In order to use the MLR and MRMT algorithms, we have used the classificatio via regression method implemented in WEKA (CVR). Thus, the selection of MLR or MRMT is a learning parameter of GA-Stacking (MLR by default).

\subsection{Learning parameters of algorithms}

In the related work on Stacking, the algorithms used by other authors to generate both the base-level classifier and the meta-classifie employ the default learning parameters. Since these parameters can influenc the results of each classifie, GA-Stacking searches in the parameter space of the learning algorithms, in addition to selecting the base and meta classifiers In Appendix A we detail the learning parameters that we have used for every learning algorithm.

\subsection{Other parameters}

In addition to the learning algorithms and their parameters, there are other aspects related to $G A$ Stacking, such as the size of the ensemble of classifiers the representation of candidate solutions and the GA parameters. In this section, we describe these parameters in more detail.

\subsubsection{Size of the ensemble of classifie $s$}

The number of algorithms that must be used to generate the level- 0 classifier varies from one study to another in the literature. For example, Ting and Witten [34] use three algorithms, whereas Seewald [32] uses six algorithms. Recently Džeroski and Ženko [14] use three and seven base classifier to carry out the comparison among different methods of ensemble construction. 


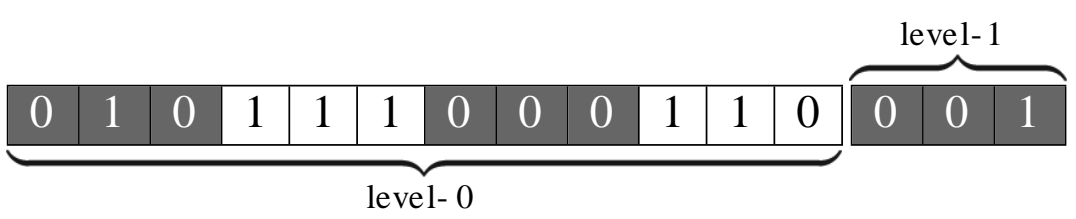

Fig. 8. Binary codificatio of the GAS5NPI configuration

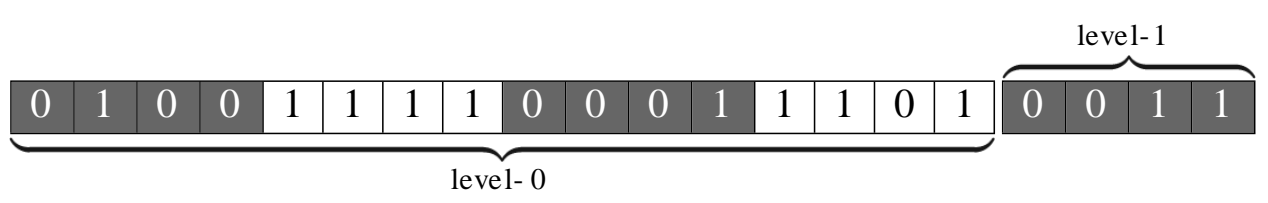

Fig. 9. Binary codificatio of the GAS5NPII configuration

Since there is no consensus regarding the number of base classifier that must be members of the ensemble of classifiers we include the ensemble size in the search space of GA-Stacking. We set "a priori" only the maximum number of base classifiers Thus, we allow in the representation of chromosomes that one or more of the places in the chromosome for the base classifier can contain the empty-classifie. In this way, the number of base-level classifier can vary from 0 to the maximum number.

In order to determine the influenc of the number of base classifier in the ensemble, the maximum number of base classifier has been set to four and ten. Therefore, we include the number of base classifier used in previous studies in the literature.

\subsubsection{Solutions representation}

As we detailed in Section 3.2, GA-Stacking uses a binary codificatio in order to represent the solutions. The solution representation depends essentially on three factors previously mentioned: the number of learning algorithms available, the use of learning parameters of algorithms and the maximum number of base classifier in the ensemble. Taking into account these factors, we have developed six GA-Stacking setups with the purpose of determining the best one of them. Next we detail the codificatio of each configuration

- GAS5NPI ${ }^{1}$. The firs GA-Stacking configuratio in these experiments is similar to the one used in previous experiments (Section 4). The only difference is that now the non-presence of algorithms (i.e. the presence of empty-classifiers in a given position of the chromosome is considered. The maximum number of possible base classifier is four $(n=4)$, and the learning parameters of the algorithms are not included in the solution. The number of algorithms available is seven $(m=7)$. Figure 8 shows the solution codificatio of this GA-Stacking configuration The number of genes in the chromosome is $T_{c}=5$, and the size in bits is $T_{b}=15$.

- GAS5NPII. This configuratio is similar to the previous one, with the only difference that now the number of available algorithms is $15(m=15)$. Figure 9 displays the solutions codificatio in this configuration

\footnotetext{
${ }^{1}$ GA-Stacking configuration names come from, GA-Stacking, "GAS", the maximum number of ensemble members, " 5 " and " 11 " (including the meta-classifier) the use or not of the learning parameters of the algorithms, "WP" (with parameters) and "NP" (no parameters), and the experiment version depending on the number of available algorithms, "I" or "II" (7 and 15 respectively).
} 


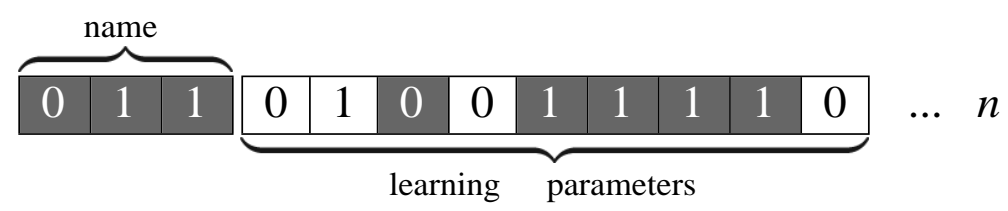

Fig. 10. A classifie binary codificatio within the GAS5WPI configuration

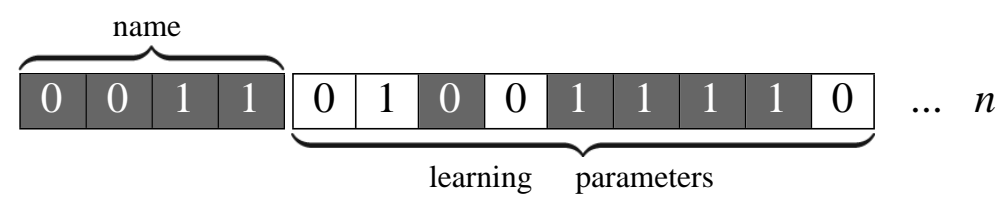

Fig. 11. A classifie binary codificatio within the GAS5WPII configuration

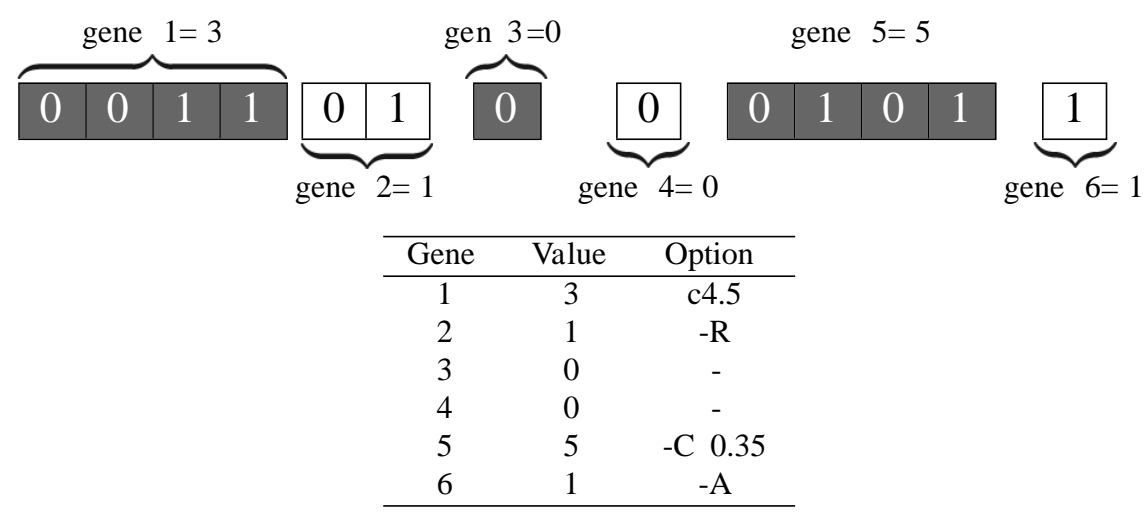

Fig. 12. The representation of $\mathrm{C} 4.5$ and its learning parameters using a binary codification

The number of genes in the chromosome is $T_{c}=5$ and the size in bits is $T_{b}=20$.

- GAS5WPI. In this GA-Stacking configuratio we included the parameters of each learning algorithm in the genetic search. In these experiments $m=7$ and $n=5$. Due to the amount of available algorithms and their parameters, we have chosen a general representation of the solutions that incorporated most of the parameters available. In Appendix B we describe the selected learning parameters of each algorithm and the gene that represents them.

Since the number and type of learning parameters varies from an algorithm to another, and there might be mutually excluding parameters for the same algorithm, we designed a general codification although this implies that in some cases some genes might not code any parameter of the learning algorithm.

Figure 10 shows the codificatio of one algorithm within the chromosome. Six genes are used to represent a classifie, one for the name and fi e to code the learning parameters. The number of genes in the chromosome is $T_{c}=30$ and the size in bits is $T_{b}=60$.

- GAS5WPII. In this configuratio $m=15$. Therefore, the size in bits of the chromosome is $T_{b}=65$ whereas the number of genes of the chromosome is $T_{c}=30$ again. Figure 11 shows the codificatio and Fig. 12 shows an example of a classifie codificatio that will be generated from C4.5.

- GAS11NP. In this GA-Stacking configuratio we extend the number of maximum base classifier in the ensemble. In this case $m=10$ and the used codificatio is similar to the one used by GAS5NPII. 
Table 7

Datasets descriptions

\begin{tabular}{lccrcc}
\hline Dataset & Attributes & Instances & Part A & Part B & Classes \\
\hline australian & 14 & 690 & 345 & 345 & 2 \\
balance & 4 & 625 & 312 & 313 & 3 \\
breast-w & 9 & 699 & 349 & 350 & 2 \\
car & 6 & 1728 & 1382 & 346 & 4 \\
chess & 36 & 3196 & 2876 & 320 & 2 \\
diabetes & 8 & 768 & 384 & 384 & 2 \\
echo & 6 & 132 & 66 & 66 & 2 \\
german & 20 & 1000 & 500 & 500 & 2 \\
glass & 9 & 214 & 107 & 107 & 6 \\
heart & 13 & 270 & 135 & 135 & 2 \\
hepatitis & 19 & 155 & 77 & 78 & 2 \\
hypo & 25 & 3163 & 2846 & 317 & 2 \\
image & 19 & 2310 & 1848 & 462 & 7 \\
ionosphere & 34 & 351 & 175 & 176 & 2 \\
iris & 4 & 150 & 75 & 75 & 3 \\
soya & 35 & 683 & 341 & 342 & 19 \\
vote & 16 & 435 & 217 & 218 & 2 \\
wine & 13 & 178 & 89 & 89 & 3 \\
\hline
\end{tabular}

Regarding the length of the chromosome, it is given by $T_{c}=11$ and $T_{b}=44$ bits.

- GAS11WP. This is the last evaluated configuratio of GA-Stacking. In this configuratio the maximum number of base-level classifier is ten, like in the previous configuration but in this case we included the learning parameters of each algorithm. The codificatio used is similar to the one used by GAS5WPII, but taking into account the new value of $m$. Regarding the chromosome length, it is given by $T_{c}=66$ and its length in bits is $T_{b}=143$.

\subsection{Experimental Setup}

In this section we describe in detail the configuratio of the experiments carried out with the purpose of evaluating the different versions of GA-Stacking.

\subsubsection{Domains}

For the following experiments we have used 18 data sets from the UCI repository. These datasets have been used widely in other stacking comparative works. In order to evaluate the GA-Stacking approach in a different set of instances than the GA training set, we divided each data set into two parts: part $A$ is used to evaluate and compare stacking approaches and part $B$ is used to fin the best stacking configuration that is, part $B$ is used during the learning process. Table 7 shows the data sets characteristics.

\subsubsection{GA parameters}

Due to the increase in size of the configuration search space caused by the increase of available learning algorithms and their parameters, we augmented the size of the population and the number of generations. The parameters used for the GA in the following experiments are shown in Table 8 .

\subsubsection{Comparison of GA-Stacking versions}

In order to evaluate the different versions of $G A$-Stacking, each configuratio was executed three times in each domain with dataset $B$. The best individual found by GA-Stacking is taken as the best configuratio of Stacking. This individual is composed of the name of the algorithms (and possibly 
Table 8

GA parameters

\begin{tabular}{lr}
\hline Parameter & Value \\
\hline Population & 50 \\
Generations & 50 \\
Elite rate & 0.10 \\
Cull rate & 0.40 \\
Mutation rate & 0.10 \\
\hline
\end{tabular}

its parameters) to be used as meta and base classifiers But the individual is not yet a proper stacking system, because these algorithms have yet to be trained. This is done with dataset $A$, as explained next. All the configuration found by the different versions of GA-Stacking are compared to each other by means of a 10 -fold stratifie cross-validation using dataset $A$. A paired t-test is used to test the statistical significanc with a confidenc of $95 \%$.

In order to compute the relative improvement obtained by the configuration of Stacking found by a given version of GA-Stacking over the rest of versions, we compute the Average Relative Improvement (ARI) for all domains used.

\subsubsection{Other parameters}

Again, we have used the learning algorithms implemented in WEKA (version 3.4). Also, in order to determine the statistical significanc of the results, we have used the paired t-test implemented in WEKA. As explained in Section 4.2, in order to avoid overfitting GA-Stacking carries out a cross-validation procedure to compute the fitnes of an individual. In the rest of experiments, a 2-fold cross-validation is used, as a trade-off between accuracy and computation time. The fitnes value is the average of the cross-validation process. Figure 13 shows the fitnes computation process.

The Stacking algorithm carries out another cross-validation-like process, in order to build the meta-level data. By default this process is a 10 -fold cross-validation.

\subsection{Empirical results}

In this section we show the results of comparing different version of GA-Stacking. The accuracy of the ensemble of classifier constructed from the Stacking configuration found by GA-Stacking can be seen in Table 9. In bold we show the best results.

Nevertheless, if we want to compare the Stacking configuration performance found by the different versions of GA-Stacking, Table 10 is more interesting: it reflect the ARI of $X$ over $Y$ for each solution pair $X$ and $Y$. Also, this table shows the win/loss summary computed from a paired t-test (10-fold cross-validation). The win/loss summary represents the number of domains where the algorithm in the row $X$ of the table is significantl better (or worse) than the algorithm in column $Y$. The column "Total" adds all the win:loss summaries in the row.

Analyzing Table 10 we can see that the difference in the ARI in all domains among different solutions found by $G A$-Stacking is low (between $-1,86 \%$ and $1,82 \%$ ). Nevertheless, if we analyze the last column, we can see that the GA-Stacking versions that include the parameters in the genetic search are better than the others. In other words, GAS5WPI $(+8)^{2}$, GAS11wP $(+7)$ and GAS5WPII $(+5)$, are better than GAS5NPI (-3), GAS5NPII (-4) y GAS11NP $(-13)$. Thus, the use of the parameters of the algorithms, by extending the search space for the GA, obtains better results than not using them.

\footnotetext{
${ }^{2}$ The number in brackets represents the absolute difference between wins and loses
} 
A. Ledezma et al. / GA-stacking: Evolutionary stacked generalization

Table 9

Results of the Stacking configuration found by different versions of GA-Stacking

\begin{tabular}{lcccccc}
\hline Domains & GAS5NPI & GAS5NPII & GAS5WPI & GAS5WPII & GAS11NP & GAS11WP \\
\hline australian & 86.93 & 87.21 & $\mathbf{8 8 . 1 1}$ & 87.22 & 88.09 & 88.08 \\
balance & 90.08 & 94.23 & $\mathbf{9 4 . 5 4}$ & 92.95 & 94.22 & 94.23 \\
breast & 95.42 & 95.71 & $\mathbf{9 7 . 1 4}$ & 95.99 & 96.57 & 96.28 \\
car & 96.02 & 95.15 & 97.03 & 97.18 & 95.88 & $\mathbf{9 7 . 4 7}$ \\
chess & 99.27 & 99.17 & 99.23 & 99.24 & $\mathbf{9 9 . 3 4}$ & 99.27 \\
diabetes & 73.41 & 73.93 & $\mathbf{7 5 . 2 4}$ & 73.93 & 74.99 & $\mathbf{7 5 . 2 4}$ \\
echo & 86.67 & 84.17 & 87.50 & $\mathbf{9 0 . 0 0}$ & 84.17 & $\mathbf{9 0 . 0 0}$ \\
german & 74.00 & $\mathbf{7 5 . 4 0}$ & 71.00 & 75.00 & 71.00 & 73.60 \\
glass & 62.45 & 63.45 & 67.27 & $\mathbf{7 6 . 0 0}$ & 66.27 & 74.55 \\
heart & 82.75 & $\mathbf{8 3 . 5 7}$ & 82.03 & 82.80 & 75.44 & 82.75 \\
hepatitis & $\mathbf{8 0 . 8 9}$ & $\mathbf{8 0 . 8 9}$ & 79.64 & 78.57 & 80.71 & 79.64 \\
hypho & $\mathbf{9 9 . 3 0}$ & 99.12 & 99.26 & 98.98 & 98.95 & 99.23 \\
images & 97.94 & 97.89 & 97.56 & $\mathbf{9 8 . 1 6}$ & 97.62 & 98.10 \\
ionosphere & 89.02 & 90.23 & $\mathbf{9 1 . 9 3}$ & 89.61 & 90.26 & 90.23 \\
iris & 93.57 & 92.32 & 93.57 & 92.32 & 94.82 & $\mathbf{9 7 . 3 2}$ \\
sonar & $\mathbf{9 7 . 1 8}$ & 94.27 & 91.36 & 93.36 & $\mathbf{9 7 . 1 8}$ & 95.27 \\
vote & $\mathbf{9 5 . 8 7}$ & 93.07 & 95.84 & $\mathbf{9 5 . 8 7}$ & 91.75 & 94.00 \\
wine & 94.44 & 96.67 & $\mathbf{9 8 . 8 9}$ & 97.78 & 94.31 & 92.22 \\
\hline
\end{tabular}

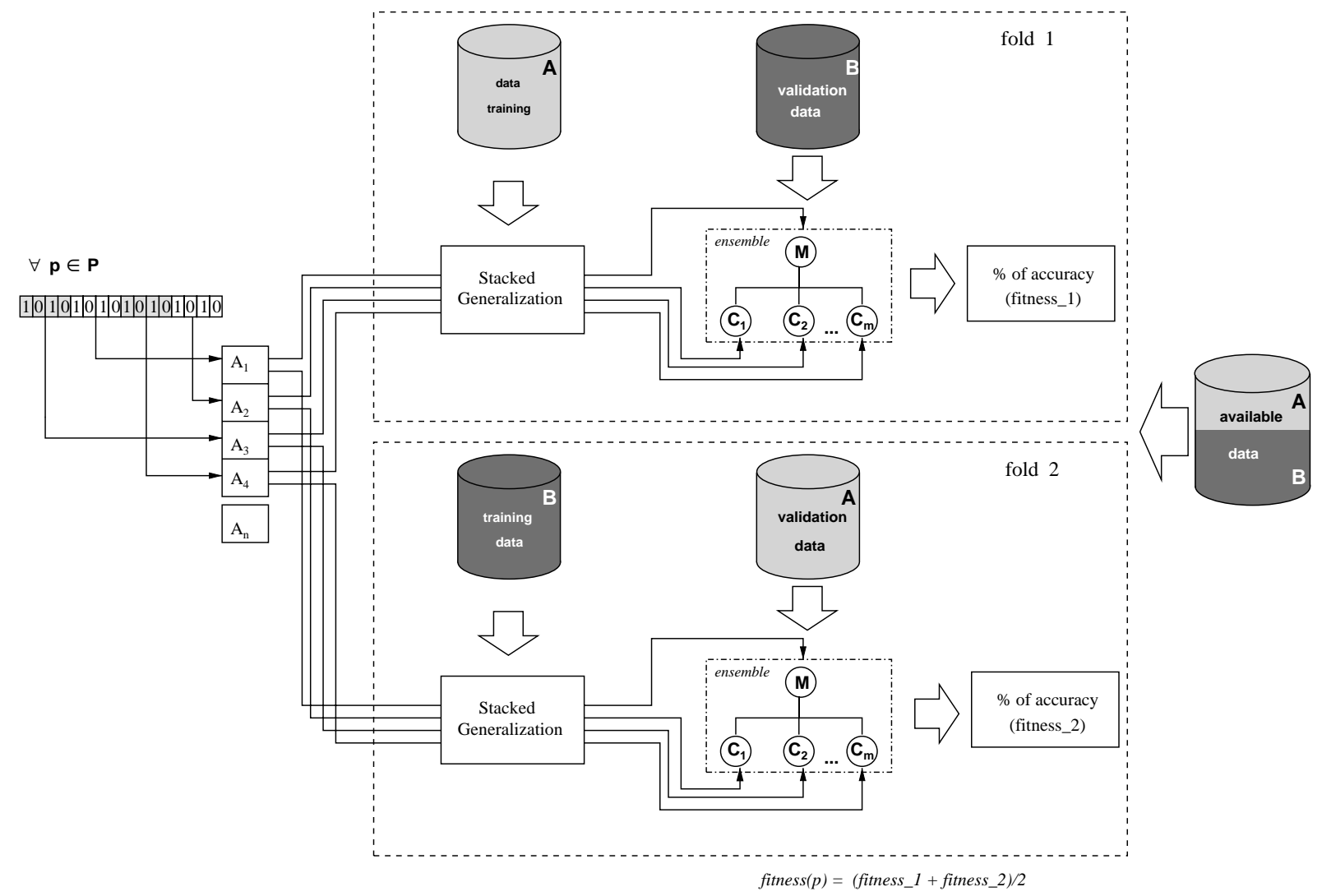

Fig. 13. Fitness computation process using a 2-fold cross-validation. 
Table 10

Relative improvement of Stacking configuration found by different versions of GA-Stacking. Elements in $X$ row and $Y$ column show the relative improvement of $X$ over $Y$ (in percentage) and the win/loss summary (according to $1 \times 10 t$-test)

\begin{tabular}{|c|c|c|c|c|c|c|c|}
\hline & GAS5NPI & GAS5NPII & GAS5WPI & GAS5WPII & GAS11NP & GAS11WP & Total \\
\hline GAS5NPI & & $0.121: 1$ & $0.801: 3$ & $1.462: 3$ & $-0.223: 1$ & $1.60 \quad 1: 3$ & $8: 11$ \\
\hline GAS & -0.1 & & $0.660: 2$ & $0: 2$ & $2: 0$ & $1.47 \quad 0: 2$ & $3: 7$ \\
\hline GAS & $-0.813: 1$ & $-0.672: 0$ & & $-0.672: 0$ & -1 & $0.81 \quad 0: 1$ & $11: 3$ \\
\hline GAS & $-1.483: 2$ & $-1.352: 0$ & $-0.670: 2$ & & $-1.714: 1$ & $0.14 \quad 2: 1$ & $11: 6$ \\
\hline GA & 0.22 & $0: 2$ & $1.021: 4$ & $1: 4$ & & $1.820: 3$ & $3: 16$ \\
\hline GAS11WP & $-1.633: 1$ & $-1.492: 0$ & $-0.821: 0$ & $-0.141: 2$ & $-1.863: 0$ & & $10: 3$ \\
\hline
\end{tabular}

Also, the increase of the number of available algorithms (I vs. II) and the increase of the maximum number of classifier in the ensemble does not seem to improve very much the solution fitness This can be explained by the significan increase in the search space, and as we carried out 50 generations of 50 individuals, the number of explored configuration is relatively small compared to the search space.

If the use of the parameters of the algorithms is combined with the increase of the number of available algorithms and the maximum number of members in the ensemble (GAS11wP/10:3), we obtain results that are comparable with the best one of the GA-Stacking versions (GAS5WPI/11:3). In other words, if we compare GAS5WPI and GAS11wP, both are significantl worse three times than another GA-Stacking configuration Nevertheless, GAS5WPI has more wins than GAS11wP (11 vs. 10), but if we compare the significan differences between both of them, GAS11wP is the best one of the two versions $(+1)$. Therefore, we select the maximal GA-Stacking version for the rest of the comparisons.

\section{GA-Stacking performance}

In order to estimate the $G A$-Stacking performance we have carried out a comparison among $G A$ Stacking (GAS11wP version) and the best generators of homogeneous ensemble of classifiers Boosting and Bagging, in addition to other algorithms for ensemble generation based on Stacking.

\subsection{Experimental setup}

In this section we detail the setup of the experiments with the purpose of comparing GA-Stacking with other methods of ensemble generation.

\subsection{Domains}

In these experiments we have used a subset of the domains used for evaluating different versions of GA-Stacking (Section 5.4.1), and also used in previous related work. The selection of these domains was carried out taking into account the number of classes, instances and attributes with the purpose of using representative domains. The selected domains are: australian, balance, car, diabetes and glass.

\subsection{GA parameters}

As we mentioned earlier, the GA-Stacking configuratio used in these experiments is GAS11WP. This GA-Stacking version can have up to 10 base-level classifier and includes the parameters of the algorithms used to generate the classifiers The GA parameters are the same than in a previous section (see Table 8). 


\subsection{Learning algorithms}

We have used two categories of algorithms in these experiments. The firs of these categories includes the algorithms for generating the individual classifiers These algorithms are used by GA-Stacking and the other algorithms for ensemble generation in order to obtain the ensemble members. The other category includes the ensemble of classifier algorithms and classifier combination methods.

The learning algorithms for generating the individual classifier are the same ones used in the $G A$ Stacking versions comparison (see Section 5.1). Regarding the ensemble generation algorithms and classifier combination methods, we have used the following ones:

- VOTE: Method for combining classifier using unweighted average of probability estimates.

- BESTCV: Method for selecting a classifie from among several ones using cross-validation on the training data.

- Bagging. The base algorithm used is C4.5.

- Boosting (AdaBoostM1) The base algorithm used is C4.5.

- SMRMT: Stacking with multi-response model trees. Given that a study uses groups of three and seven algorithms to generate the base-level classifier [14], we have used two versions of this algorithm. The firs group is composed by C4.5, IBk and Naive Bayes, while we add to the second group $K^{*}$, Decision Table, MLR and Kernel Density. In [14] a comparative among the ensemble construction methods based on Stacking was carried out. They concluded that SMRMT has a greater performance than any of the other Stacking based methods.

- SCMLR (StackingC): Stacking with a reduced number of meta-level attributes independently of the number of classes. We have used three groups of algorithms to generate the base-level classifiers First, we have used the classifier used by Seewald [32] (Decision Table, C4.5, Naive Bayes, Kernel Density, $M L R$ and $K^{*}$ ). Second, we have used the groups used by D žeroski y Ženko [14] described in the previous item.

- SMLR: Stacking with multi-response linear regression proposed by Ting y Witten [34]. Like in the other Stacking-based algorithms we have used the groups of three and seven algorithms previously described.

\subsection{Comparing results}

In order to evaluate and compare results of different algorithms, we estimated the classificatio accuracy with a stratifie ten-fold cross-validation. In order to compare the results obtained by GA-Stacking with each one of the used algorithms, we have computed the relative improvement of GA-Stacking over other algorithms in each domain. In addition, a paired t-test has been carried out $(1 \times 10)$ with the purpose of determining if the improvement obtained by GA-Stacking is statistically significant

\subsection{Experimental results}

The accuracy percentage of the different learning algorithms used in these experiments are reflecte in Table 11. As it can be seen, the results obtained by the Stacking configuration found by GA-Stacking are better in four out of the fi e domains. Also, in order to compare the GA-Stacking performance with each one of the used algorithms, in Table 12 we show the relative improvement that obtains GA-Stacking when comparing it with the other algorithms and their statistical significanc based on a paired t-test. The results will be analyzed in more detail next. 
Table 11

Accuracy rate (\%) of ensemble-generation methods and combining-classifier methods

\begin{tabular}{lccccc}
\hline Algorithms & australian & balance & car & diabetes & glass \\
\hline GAS11WP & $\mathbf{8 7 . 1 0}$ & $\mathbf{9 6 . 9 6}$ & $\mathbf{9 9 . 4 2}$ & 75.25 & $\mathbf{7 8 . 9 4}$ \\
Boosting & 83.91 & 79.21 & 96.18 & 71.87 & 73.33 \\
Bagging & 85.80 & 82.25 & 93.29 & 76.30 & 72.90 \\
BESTCV & 82.32 & 86.88 & 93.75 & 70.94 & 70.50 \\
VOTE & 86.96 & 86.88 & 94.85 & 76.56 & 76.62 \\
SMRMT3 & 85.65 & 92.97 & 99.13 & 76.02 & 68.18 \\
SMRMT7 & 85.80 & 92.50 & 98.96 & 76.29 & 73.74 \\
SCMLR5 & 85.94 & 92.50 & 95.02 & $\mathbf{7 7 . 0 7}$ & 76.08 \\
SCMLR3 & 85.51 & 90.56 & 93.92 & 76.15 & 70.11 \\
SCMLR7 & 85.65 & 89.92 & 95.02 & 76.94 & 76.56 \\
SMLR3 & 85.94 & 89.92 & 94.73 & 76.16 & 66.75 \\
SMLR7 & 85.51 & 89.28 & 95.49 & 76.94 & 76.06 \\
\hline
\end{tabular}

Table 12

Relative improvement (\%) of GA-Stacking over other ensemble and combined methods. $+/-$ means better/worse, '.' means there is no significan difference

\begin{tabular}{lcccccc}
\hline Algorithms & australian & balance & car & diabetes & glass & MRI $^{3}$ win/loss \\
\hline Boosting & $19.82+$ & $85.40+$ & $84.84+$ & $12.00+$ & 21.02. & $58.484+/ 0-$ \\
Bagging & 9.18. & $82.90+$ & $91.38+$ & -4.43. & $22.28+$ & $59.523+/ 0-$ \\
BESTCV & $27.05+$ & $76.85+$ & $90.74+$ & $14.82+$ & $28.61+$ & $60.585+/ 0-$ \\
VOTE & 1.11. & $76.86+$ & $88.76+$ & -5.58. & 9.91. & $52.392+/ 0-$ \\
SMRMT3 & 10.10. & $56.83+$ & 33.35. & -3.22. & $33.81+$ & $29.292+/ 0-$ \\
SMRMT7 & $9.18+$ & $59.51+$ & 44.45. & -4.37. & $19.80+$ & $29.763+/ 0-$ \\
SCMLR5 & 8.25. & $69.88+$ & $88.37+$ & -7.95. & 11.95. & $50.222+/ 0-$ \\
SCMLR3 & $11.00+$ & $67.82+$ & $90.47+$ & -3.77. & $29.54+$ & $54.294+0-$ \\
SCMLR7 & 10.10. & $69.88+$ & $88.37+$ & -7.31. & 10.16. & $50.282+/ 0-$ \\
SMLR3 & 8.25. & $69.87+$ & $89.01+$ & -3.80. & $36.65+$ & $54.283+/ 0-$ \\
SMLR7 & $11.00+$ & $71.67+$ & $87.17+$ & -7.34. & 12.03. & $50.233+/ 0-$ \\
\hline
\end{tabular}

\subsubsection{Analysis}

First we will analyze the results of GA-Stacking comparing it with the homogeneous ensembleconstruction methods Bagging and Boosting. As we can see in Table 12, in four of the fi e used domains GA-Stacking is significantl better than Boosting. In the case of Bagging, GA-Stacking is significantl better in three of the fi e domains. The relative improvement in both cases surpasses $58 \%$.

When comparing GA-Stacking with the combination methods by votes (Vote) and the best classifie selection by cross-validation (BestCV), we have also obtained different results. If we compare $G A$ Stacking with BestCV, GA-Stacking is significantl better in all domains with a relative improvement over $60 \%$. Nevertheless, when comparing it with the voting scheme, the significan improvements are reduced to two of the fi e domains, but the ARI continues to be above $52 \%$.

Now we will analyze the results of comparing GA-Stacking with other Stacking-based algorithms (SMRMT, SCMLR, SMLR). First, when comparing GA-Stacking with the two versions of SMRMT (three and seven base classifiers) we can observe that the number of times in which GA-Stacking is significantl better varies according to the number of base-level classifier that are members of the ensembles generated by SMRMT. GA-Stacking wins one more time if it compares with SMRMT7 instead of with SMRMT3. And the relative improvement in both is over $29 \%$.

\footnotetext{
${ }^{3}$ Mean of relative improvement.
} 
Table 13

Average number of base classifier in the solutions found by $G A$ Stacking

\begin{tabular}{lc}
\hline Domain & \# of base classifier \\
\hline australian & 9.3 \\
balance & 9.4 \\
car & 9.5 \\
diabetes & 9.4 \\
glass & 9.6 \\
\hline
\end{tabular}

In relation to the comparisons with the three versions of SCMLR (three, fi e and seven base classifiers) the number of domains in which GA-Stacking is significantl better are similar in the SCMLR5 and SCMLR7 versions $(2+)$. Nevertheless, the number of domains in which GA-Stacking is significantl better doubles when compared with the version of only three base classifiers The ARI in all cases is over $50 \%$.

Finally, if we compare the results of GA-Stacking with the results obtained by SMLR in its two versions (three and seven base classifiers) the ARI is higher than 50\%. In addition in three of the fi e domains, GA-Stacking is significantl better than SMLR.

These results demonstrate that the number of base classifier influenc the accuracy, as also does the meta-classifie employed. Given that GA-Stacking can automatically select these, among other parameters, and do not incur in overfitting it has an advantage over the manually preconfigure systems. Also, as we can see in the last row of Table 12, GA-Stacking is not significantl worse than any other algorithm in any domain.

\subsubsection{Description of the solutions}

With the purpose of observing the structure of the Stacking configuration found by GA-Stacking, we have analyzed the best individuals of each fold in the stratifie cross-validation. That is to say, of the three executions of GA-Stacking in each fold of the cross-validation, we have analyzed the individual with the greatest value in the fitnes function.

Table 13 shows the average number of base classifier in the solutions. As we can see, the number of base classifier varies between 9 and 10 , where 10 is the maximum number of base classifier that allows the GAS11wP GA-Stacking configuration On the one hand, it should be expected that the GA tends to use as many base-classifier as possible, because the probability of the empty classifie is very small, so there is a natural bias towards complex configurations However, in this case, this bias has no negative influenc on the overall accuracy, because this GA-Stacking configuratio is the best one, in spite of it allowing more base-classsifier than the rest of GA-Stacking configurations

Regarding the algorithms automatically selected by GA-Stacking to generate the base-level classifiers Fig. 14 shows the number of folds out of 10 (and above 6), where a particular classifie appears as a base classifie in the optimal configuration As it can be seen, in each domain there are between three and four algorithms that are present at least in 7 of the 10 folds of the cross-validation. For instance, in the car domain, there are three base classifier (K*, MRMT, and PART) that GA-Stacking considers to be necessary for that domain, and this fact is automatically determined by our system.

Figure 15 shows the algorithms selected to be the meta-classifie in each domain. As it can be seen, the best individuals of each fold tend to use the same algorithm to generate the level-1 classifie. For example, in the balance and car domains, the algorithm selected to generate the meta-level classifie in all cross-validation folds is the Random Forest, while in the australian domain NaiveBayes is the 


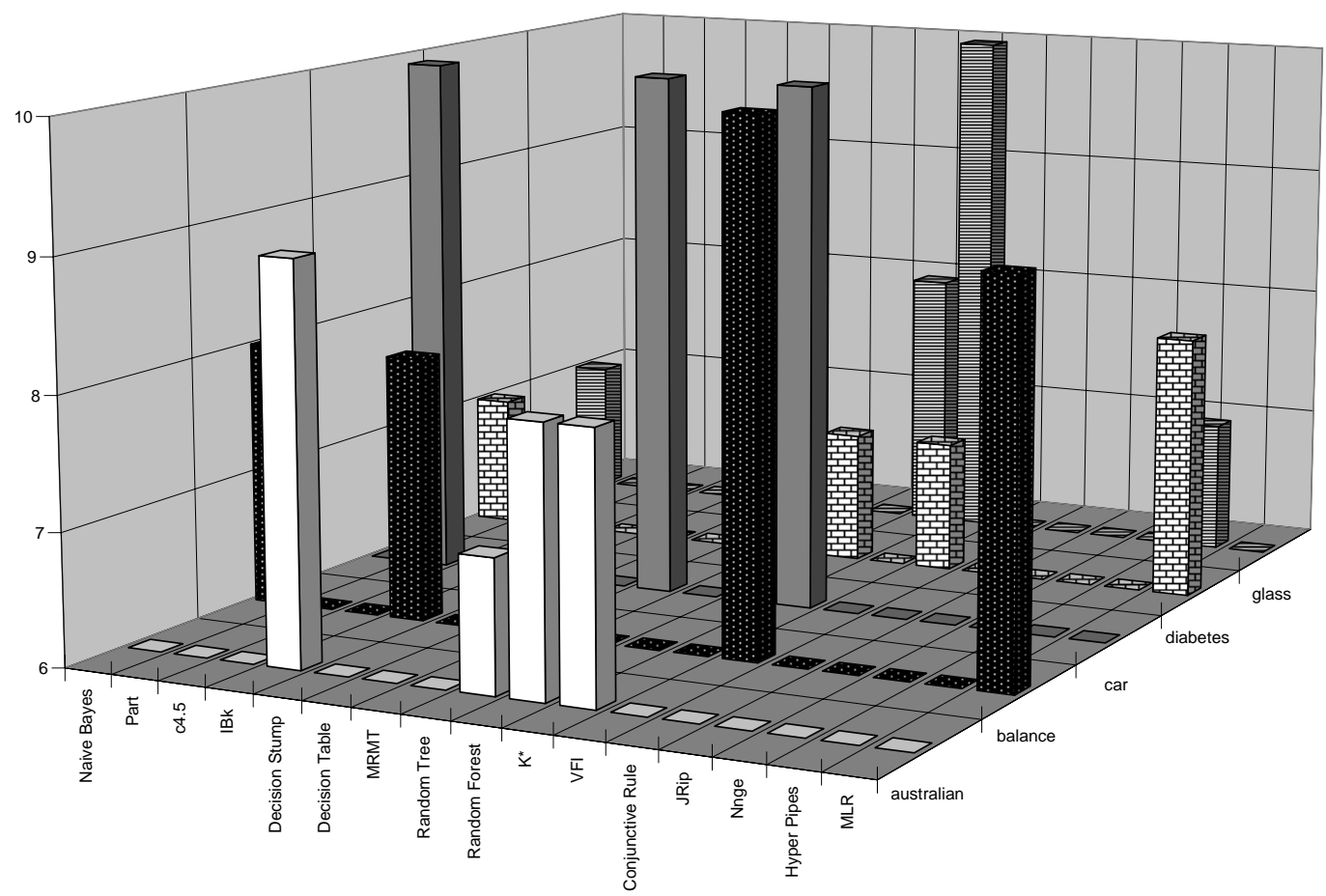

Fig. 14. Number of folds (six or more) in the cross-validation in which a specifi classifie appears in each domain.

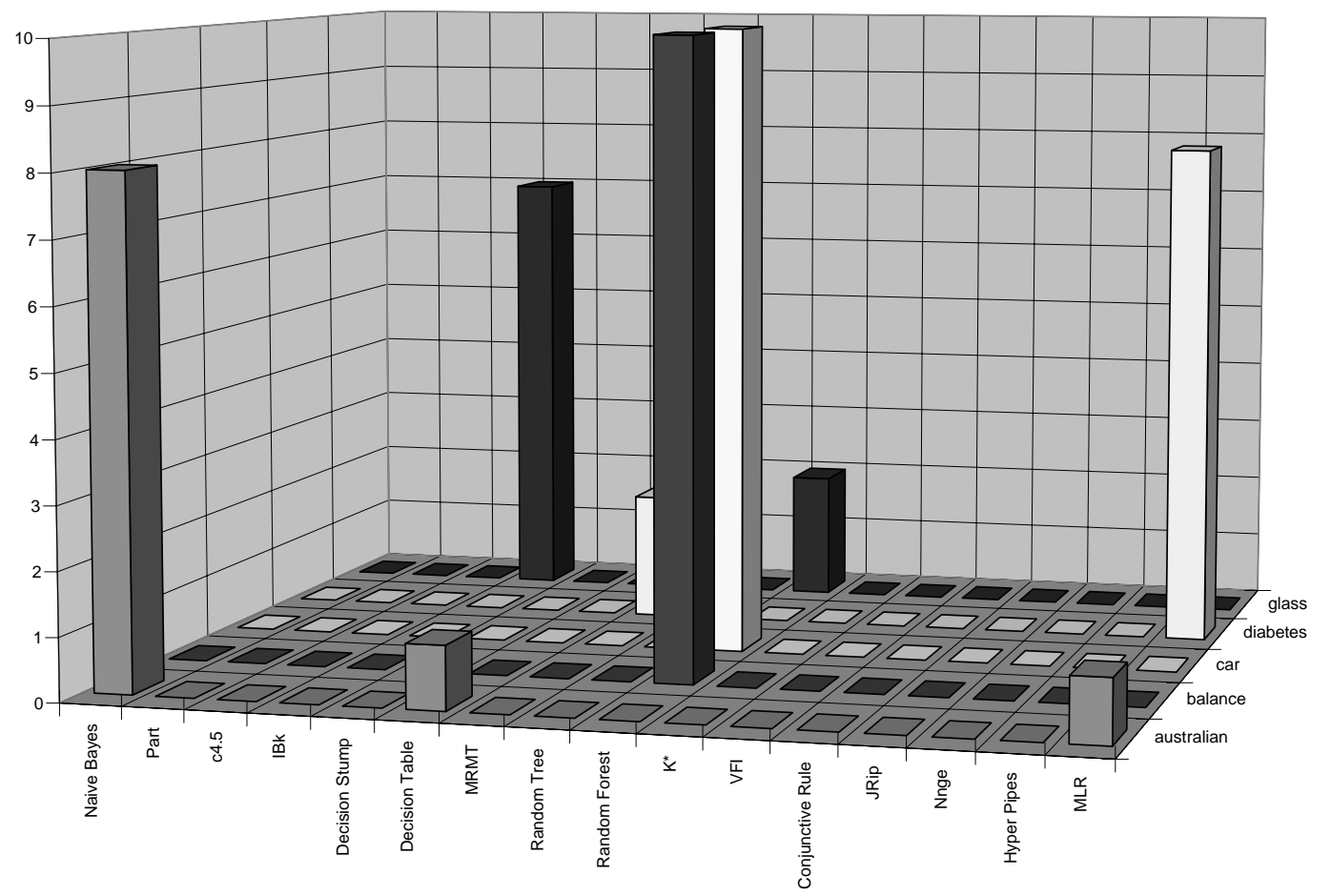

Fig. 15. Number of cross-validation folds in which a particular algorithm appears at the meta-level. 
preferred option most of the times. Other researchers claim that MLR [34,32] or MRMT [13] yield good results when used to build the meta-classifie regardless of the base algorithms and the domain. However, the algorithms selected by GA-Stacking to generate the meta-classifie vary according to the domain. For example, Naive Bayes (8/10) for australian, Random Forest (10/10) for car and balance domains, MLR $(8 / 10)$ in diabetes and $I b k(7 / 10)$ in glass.

This shows two key aspects: in each domain, a specifi algorithm is preferred over the rest to be the meta-classifier and this clearly highlights the advantage of GA-Stacking over the rest of algorithms in that it can select automatically the best algorithm for each domain.

\subsubsection{Evolution of the fitnes}

Another aspect to take into account in these experiments is the behavior of the fitnes function. In Fig. 16 we can see the evolution of the fitnes function for each one of the used domains.

The fitnes behavior is very similar in all domains: the largest increase takes place in the firs generations and soon it stays in constant growth, even when arriving at the last generation. This indicates an evolution in the solutions found by GA-Stacking. In addition, given that fitnes keeps increasing, it would be useful to increase the number of generations for the purpose of findin better individuals.

\section{Conclusions and future work}

In this paper, we have presented the GA-Stacking algorithm, an approach to fin good Stacking configuration by means of genetic search. GA-stacking not only determines which meta-level, and which (and how many) base classifier must be present, but also their parameters. The main advantage of GA-Stacking over other techniques is its fl xibility and extensibility. It can easily incorporate new learning algorithms, and it is not restricted by "a priori" assumptions. Another advantage of GA-Stacking is that the solutions that it find are domain dependent. Thus, GA-Stacking adapts the Stacking configuratio to the domain biases and characteristics, while all the other approaches use the same Stacking configuration independently from the domain in which they are applied.

We would like to highlight that in addition to GA-Stacking being able to fin accurate Stacking configurations it also provides some automation of the data mining process. Typically, Stacking has to be configure by hand. Even though there are some general guidelines on how to build Stacking configurations in some cases a process of trial and error is required from the user for selecting the base and the meta learning classifiers and also their parameters. With GA-Stacking, the intervention of the user is reduced to selecting the set of available classifiers Our experiments show that even if this set is large, results do not degrade.

Empirical results in domains currently used in this fiel show that GA-Stacking is comparable to the best results reported so far, and it is never significantl worse than the other tested systems (with the advantage that paremeters such as the number of base classifiers or the algorithms available to be used as base, need not be specifie in advance). With respect to accuracy, if we add the relative improvements over the other systems across all the domains, positive differences are always obtained, quite large in some cases. Therefore, if accuracy is very important for a given task, we believe GA-Stacking should be used.

However, GA-Stacking requires a longer execution time than the rest of approaches, because several generations of individuals must be evaluated in order to obtain a good individual. Even if for most domains this is not crucial, given that most classificatio tasks do not require to work in real time, it could be relevant for others. Also, adding incremental capabilities can be hard to implement. Finally, 
A. Ledezma et al. / GA-stacking: Evolutionary stacked generalization
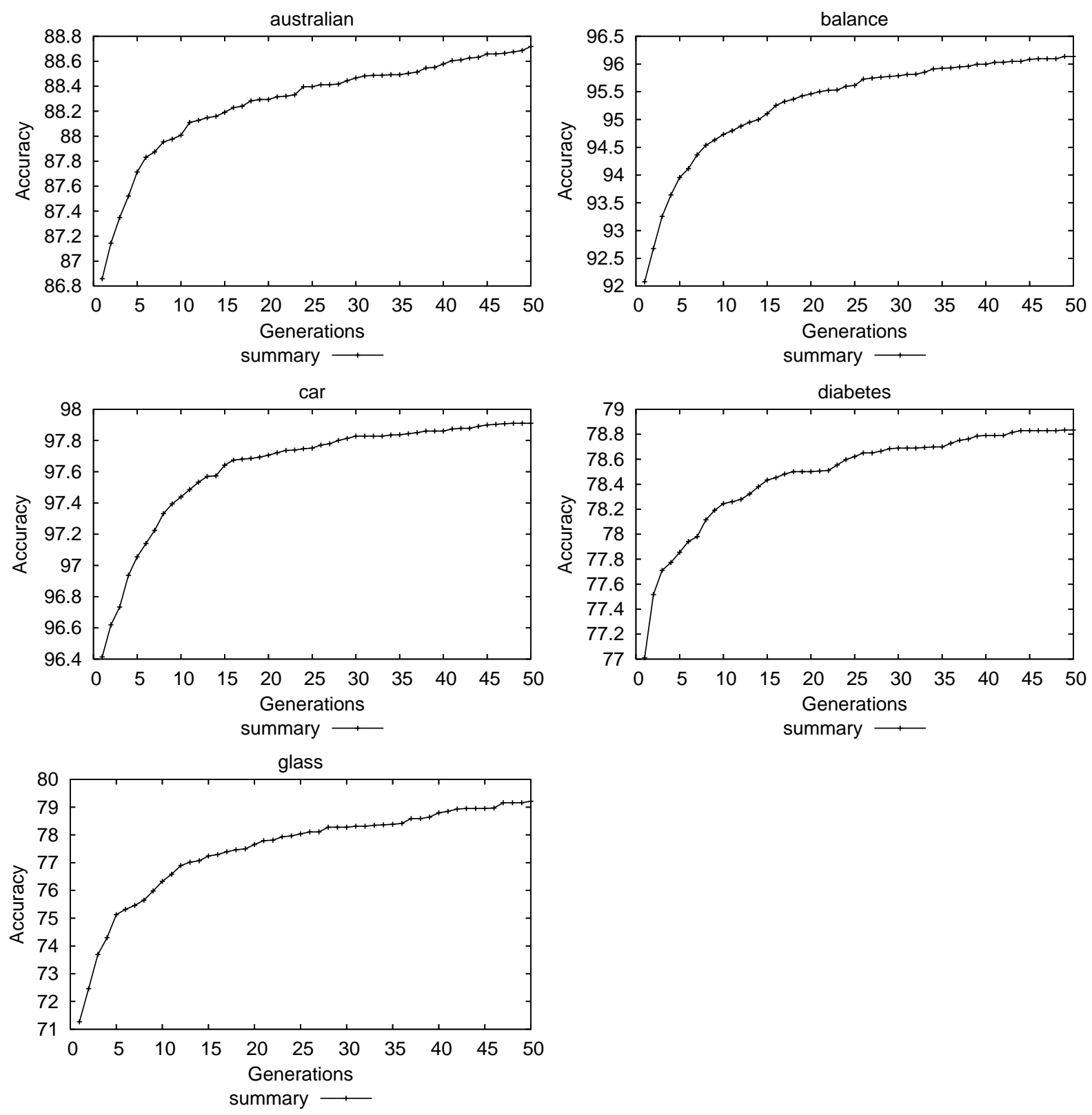

Fig. 16. Evolution of the fitnes function in each domain.

another drawback common to all Stacking research is the understandability by the human of the fina classifie scheme.

Accuracy is not always the only useful feature to consider, although it is usually the only aspect considered in Stacking research. Configuratio size, on-line classificatio speed, etc., can also be relevant issues in some domains. In the future, we plan to investigate the $\mathrm{fl}$ xibility of GA-Stacking so that these issues are also considered. For instance, we intend to add selective pressure towards accurate, but also simple (few base classifiers Stacking configurations Also, we would like to add information 
to the chromosome about the meta-level data to be used, so that the Stacking configuratio can use the most appropriate representation for each domain.

\section{Acknowledgements}

This work has been partially supported by the Spanish MCyT under projects TRA2007-67374-C02-02 and TIN-2005-08818-C04. Also, it has been supported under MEC grant by TIN2005-08945-C06-05. We thank anonymous reviewers for their helpful comments.

\section{Appendices A Parameters of the algorithms}

We present next the parameters of the learning algorithms used by GA-Stacking in order to generate the ensemble of classifier (See Table 14.)

Table 14

\begin{tabular}{|c|c|c|}
\hline Algorithm & Option & Description \\
\hline Algorithm & Option & Description \\
\hline Naive Bayes & $-\mathrm{K}$ & $\begin{array}{l}\text { Use a kernel estimator for numeric attributes rather than a normal } \\
\text { distribution. }\end{array}$ \\
\hline \multirow[t]{2}{*}{ PART } & $-\mathrm{B}$ & $\begin{array}{l}\text { Use binary splits on nominal attributes when building the partial } \\
\text { trees. }\end{array}$ \\
\hline & $-\mathrm{C}$ & Set the confidenc factor used for pruning (default: 0.25 ). \\
\hline \multirow[t]{6}{*}{$\mathrm{C} 4.5$} & $-\mathrm{R}$ & Use reduced error pruning. No subtree raising is performed. \\
\hline & $-\mathrm{U}$ & Use unpruned tree. \\
\hline & $-\mathrm{B}$ & Use binary splits for nominal attributes. \\
\hline & $-\mathrm{S}$ & Do not perform subtree raising. \\
\hline & $-\mathrm{C}$ & Set confidenc threshold for pruning. (Default: 0.25 ). \\
\hline & $-\mathrm{A}$ & If set, Laplace smoothing is used for predicted probabilites. \\
\hline \multirow[t]{5}{*}{ IB $k$} & $-\mathrm{F}$ & $\begin{array}{l}\text { Neighbors will be weighted by their similarity when voting. } \\
\text { (default equal weighting). }\end{array}$ \\
\hline & $-\mathrm{D}$ & $\begin{array}{l}\text { Neighbors will be weighted by the inverse of their distance when } \\
\text { voting. (default equal weighting) }\end{array}$ \\
\hline & $-\mathrm{N}$ & Turns off normalization. \\
\hline & $-\mathrm{S}$ & $\begin{array}{l}\text { When } K \text { is selected by cross-validation for numeric class at- } \\
\text { tributes, minimize mean-squared error. (default mean absolute } \\
\text { error) }\end{array}$ \\
\hline & $-\mathrm{K}$ & $\begin{array}{l}\text { Set the number of nearest neighbors to use in prediction (default } \\
\text { 1). }\end{array}$ \\
\hline Decision Stump & - & It does not have configurabl parameters. \\
\hline Decision Table & $-\mathrm{I}$ & Use nearest neighbor instead of global table majority. \\
\hline Classificatio by Regression & $-\mathrm{W}$ & $\begin{array}{l}\text { Specify the name of a numeric predictor as the basis for the } \\
\text { classifie. }\end{array}$ \\
\hline \multirow[t]{2}{*}{ Random Tree } & $-\mathrm{S}$ & $\begin{array}{l}\text { Sets the random number seed used for selecting attributes. (de- } \\
\text { fault: 1). }\end{array}$ \\
\hline & $-\mathrm{K}$ & Sets the number of randomly chosen attributes at each node. \\
\hline \multirow[t]{2}{*}{ Random Forest } & $\begin{array}{l}-\mathrm{M} \\
-\mathrm{I}\end{array}$ & Set the number of trees in the forest (default 10). \\
\hline & $-\mathrm{K}$ & $\begin{array}{l}\text { Set the number of features to consider. If }<1 \text { (the default) will } \\
\text { use } \log M+1 \text {, where } M \text { is the number of inputs. }\end{array}$ \\
\hline
\end{tabular}




\begin{tabular}{|c|c|c|}
\hline Algorithm & Option & Description \\
\hline \multirow{4}{*}{$\mathrm{K}^{*}$} & $-\mathrm{S}$ & Random number seed (default 1 ). \\
\hline & $-\mathrm{B}$ & Manual blend setting (default 20\%). \\
\hline & $-\mathrm{E}$ & Enable entropic auto-blend setting. \\
\hline & $-\mathrm{M}$ & Specify the missing value treatment mode (default average). \\
\hline \multirow[t]{2}{*}{ VFI } & $-\mathrm{B}$ & Set exponential bias towards confiden intervals. (default 1.0). \\
\hline & $-\mathrm{C}$ & Do not weight voting intervals by confidence \\
\hline \multirow[t]{5}{*}{ Conjunctive Rule } & $-\mathrm{S}$ & Set the seed of randomization (default 1 ). \\
\hline & $-\mathrm{R}$ & Set if NOT uses randomization (default:use randomization). \\
\hline & $-\mathrm{E}$ & $\begin{array}{l}\text { Set whether consider the exclusive expressions for nominal at- } \\
\text { tributes (default false). }\end{array}$ \\
\hline & $-\mathrm{N}$ & $\begin{array}{l}\text { Set number of folds for REP One fold is used as pruning set. } \\
\text { (default 3). }\end{array}$ \\
\hline & $-\mathrm{M}$ & $\begin{array}{l}\text { Set the minimal weights of instances within a split. (default } \\
2.0 \text { ). }\end{array}$ \\
\hline \multirow{5}{*}{ JRIP } & $-\mathrm{O}$ & Set the number of runs of optimizations. (Default: 2 ). \\
\hline & $-\mathrm{P}$ & Whether NOT use pruning (default: use pruning). \\
\hline & $-\mathrm{E}$ & $\begin{array}{l}\text { Whether NOT check the error rate }>=0.5 \text { in stopping criteria } \\
\text { (default: check). }\end{array}$ \\
\hline & $-\mathrm{F}$ & $\begin{array}{l}\text { Set number of folds for REP One fold is used as pruning set. } \\
\text { (default 3). }\end{array}$ \\
\hline & $-\mathrm{N}$ & $\begin{array}{l}\text { Set the minimal weights of instances within a split. (default } \\
2.0 \text { ). }\end{array}$ \\
\hline \multirow[t]{2}{*}{ NNGE } & $-\mathrm{G}$ & Set the number of attempts of generalization (default 5 ). \\
\hline & $-\mathrm{I}$ & $\begin{array}{l}\text { Set the number of folders to use in the computing of the mutual } \\
\text { information (default } 5 \text { ). }\end{array}$ \\
\hline Hyper Pipes & - & It does not have configurabl parameters. \\
\hline
\end{tabular}

\section{Appendices B Genetic representation of the learning parameters}

Next, we present the correspondence between the parameters of the algorithms used by GA-Stacking and the gene that represents them within the binary codificatio (See Table 15).

Table 15

\begin{tabular}{|c|c|c|c|c|c|c|c|c|c|c|c|c|c|c|c|c|c|}
\hline Algorithm $^{4}$ & & $\mathrm{NA}^{5}$ & $\mathrm{NB}$ & PART & $\mathrm{C} 4.5$ & $\mathrm{IB} k$ & Ds & DT & $\mathrm{CPR}^{6}$ & RT & $\mathrm{RF}$ & $\mathrm{K}^{*}$ & VFI & $\mathrm{CR}$ & JRIP & NNGE & $\mathrm{HP}$ \\
\hline & Valor & 0 & 1 & 2 & 3 & 4 & 5 & 6 & 7 & 8 & 9 & 10 & 11 & 12 & 13 & 14 & 15 \\
\hline \multirow[t]{4}{*}{ Gene \# 2} & 0 & - & - & - & - & - & - & - & - & - & - & - & - & - & - & - & - \\
\hline & 1 & - & - & - & $-\mathrm{R}$ & $-F$ & - & - & - & -S 1 & -S 1 & -B 10 & -B 2.0 & $-\mathrm{S} 2$ & $-\mathrm{O} 3$ & $-G 2$ & - \\
\hline & 2 & - & - & - & $-U$ & $-D$ & - & - & - & -S 2 & $-\mathrm{S} 2$ & -B 30 & -B 3.0 & -S 3 & $-\mathrm{O} 4$ & $-G 3$ & - \\
\hline & 3 & - & - & - & - & - & - & - & - & -S 3 & -S 3 & -B 40 & -B 4.0 & -S 4 & $-\mathrm{O} 5$ & $-G 4$ & - \\
\hline \multirow[t]{2}{*}{ Gene \# 3} & 0 & - & - & - & - & - & - & - & $-\mathrm{W} A$ & - & - & - & - & - & - & - & - \\
\hline & 1 & - & $-K$ & $-B$ & $-B$ & $-\mathrm{N}$ & - & $-\mathrm{I}$ & -W B & -K 10 & -K 10 & $-E$ & $-\mathrm{C}$ & $-\mathrm{R}$ & $-P$ & - & - \\
\hline \multirow[t]{2}{*}{ Gene \# 4} & 0 & - & - & - & - & - & - & - & - & - & - & - & - & - & - & - & - \\
\hline & 1 & - & - & - & $-\mathrm{S}$ & $-S$ & - & - & - & - & - & - & - & $-E$ & $-E$ & - & - \\
\hline \multirow[t]{3}{*}{ Gene \# 5} & 0 & - & - & $-\mathrm{C} 0.10$ & $-\mathrm{C} 0.10$ & $-\mathrm{K} 1$ & - & - & - & - & - & - & - & - & - & - & - \\
\hline & 1 & - & - & -C 0.15 & $-\mathrm{C} 0.15$ & $-\mathrm{K} 2$ & - & - & - & -K 5 & $-\mathrm{I} 2$ & $-\mathrm{M} \mathrm{m}$ & - & $-\mathrm{N} 4$ & -F 4 & -I 2 & - \\
\hline & 2 & - & - & -C 0.20 & $-\mathrm{C} 0.20$ & - K 3 & - & - & - & -K 10 & -I 4 & $-\mathrm{M} \mathrm{n}$ & - & -N 5 & -F 5 & -I 3 & - \\
\hline
\end{tabular}

\footnotetext{
${ }^{4}$ It is represented by Gene \#1.

${ }^{5}$ No Algorithm.

${ }^{6}$ The gene $\# 3$ options $-A \mathrm{y}-B$ correspond to use MLR and MRMT respectively.
} 
Table 15 Continue.

\begin{tabular}{|c|c|c|c|c|c|c|c|c|c|c|c|c|c|c|c|c|c|}
\hline \multirow[t]{2}{*}{ Algorithm } & \multirow[b]{2}{*}{ Valor } & \multirow{2}{*}{$\begin{array}{c}\text { NA } \\
0\end{array}$} & \multirow{2}{*}{$\begin{array}{c}\text { NB } \\
1 \\
\end{array}$} & \multirow{2}{*}{$\begin{array}{c}\text { PART } \\
2 \\
\end{array}$} & \multirow{2}{*}{$\begin{array}{c}\text { C4.5 } \\
3\end{array}$} & \multirow{2}{*}{$\begin{array}{c}\text { IB } k \\
4\end{array}$} & \multirow{2}{*}{$\begin{array}{c}\text { Ds } \\
5 \\
\end{array}$} & \multirow{2}{*}{$\begin{array}{c}\text { DT } \\
6 \\
\end{array}$} & \multirow{2}{*}{$\begin{array}{c}\mathrm{CPR} \\
7\end{array}$} & \multirow{2}{*}{$\begin{array}{c}\text { RT } \\
8\end{array}$} & \multirow{2}{*}{$\begin{array}{c}\text { RF } \\
9\end{array}$} & \multirow{2}{*}{$\begin{array}{c}\mathrm{K}^{*} \\
10\end{array}$} & \multirow{2}{*}{$\begin{array}{c}\text { VFI } \\
11\end{array}$} & \multirow{2}{*}{$\begin{array}{r}\mathrm{CR} \\
12\end{array}$} & \multirow{2}{*}{$\begin{array}{c}\text { JRIP } \\
13\end{array}$} & \multirow{2}{*}{$\begin{array}{c}\text { NNGE } \\
14\end{array}$} & \multirow{2}{*}{$\begin{array}{r}\mathrm{HP} \\
15 \\
\end{array}$} \\
\hline & & & & & & & & & & & & & & & & & \\
\hline & 3 & - & - & $-\mathrm{C} 0.25$ & $-\mathrm{C} 0.25$ & $-\mathrm{K} 4$ & - & - & - & -K 15 & -I 6 & $-\mathrm{M} \mathrm{a}$ & - & $-\mathrm{N} 6$ & -F 6 & -I 4 & - \\
\hline & 4 & - & - & $-\mathrm{C} 0.30$ & $-\mathrm{C} 0.30$ & $-\mathrm{K} 5$ & - & - & - & -K 20 & -I 8 & $-\mathrm{M} \mathrm{d}$ & - & -N 7 & -F 7 & -I 5 & - \\
\hline & 5 & - & - & -C 0.35 & -C 0.35 & - K 6 & - & - & - & -K 25 & -I 10 & - & - & -N 8 & $-F 8$ & -I 6 & - \\
\hline & 6 & - & - & -C 0.40 & $-\mathrm{C} 0.40$ & $-\mathrm{K} 7$ & - & - & - & -K 30 & -I 12 & - & - & -N 9 & -F 9 & -I 7 & - \\
\hline & 7 & - & - & -C 0.45 & -C 0.45 & $-\mathrm{K} 8$ & - & - & - & -K 35 & -I 14 & - & - & -N 10 & -F 10 & -I 8 & - \\
\hline & 8 & - & - & -C 0.50 & $-\mathrm{C} 0.50$ & $-\mathrm{K} 9$ & - & - & - & $-\mathrm{K} 40$ & -I 16 & - & - & - & - & -I 9 & - \\
\hline & 9 & - & - & -C 0.50 & $-\mathrm{C} 0.50$ & -K 10 & - & - & - & -K 45 & -I 18 & - & - & - & - & -I 10 & - \\
\hline & 10 & - & - & -C 0.50 & $-\mathrm{C} 0.50$ & $-\mathrm{K} 11$ & - & - & - & -K 50 & -I 20 & - & - & - & - & - & - \\
\hline & 11 & - & - & -C 0.50 & $-\mathrm{C} 0.50$ & -K 12 & - & - & - & -K 55 & -I 22 & - & - & - & - & - & - \\
\hline & 12 & - & - & -C 0.50 & $-\mathrm{C} 0.50$ & -K 13 & - & - & - & $-\mathrm{K} 60$ & -I 24 & - & - & - & - & - & - \\
\hline & 13 & - & - & -C 0.50 & $-\mathrm{C} 0.50$ & -K 14 & - & - & - & -K 65 & -I 26 & - & - & - & - & - & - \\
\hline & 14 & - & - & $-\mathrm{C} 0.50$ & $-\mathrm{C} 0.50$ & -K 15 & - & - & - & -K 70 & -I 28 & - & - & - & - & - & - \\
\hline & 15 & - & - & $-\mathrm{C} 0.50$ & $-\mathrm{C} 0.50$ & -K 16 & - & - & - & $-K 75$ & -I 30 & - & - & - & - & - & - \\
\hline \multirow[t]{2}{*}{ Gene \# 6} & 0 & - & - & - & - & - & - & - & - & - & - & - & - & - & - & - & - \\
\hline & 1 & - & - & - & $-A$ & - & - & - & - & -M 5 & - & - & - & $-\mathrm{M} 3$ & $-\mathrm{N} 3$ & - & - \\
\hline
\end{tabular}

\section{References}

[1] D. Aha, D. Kibler and M. Albert, Instance-based learning algorithms, Mach Learn 6(1) (Jan 1991), 37-66.

[2] C. Blake and C. Merz, UCI repository of machine learning databases. databases http://www.ics.uci.edu/ mlearn/ MLRepository html, 1998.

[3] L. Breiman, Bagging predictors, Mach Learn 24(2) (1996), 123-140.

[4] L. Breiman, Random forests, Mach Learn 45(1) (2001), 5-32.

[5] K. Cherkauer, Human expert-level performance on a scientifi image analysis task by a system using combined artificia neural networks, in: Working Notes of the AAAI Workshop on Integrating Multiple Learned Models, 1996, pp. 15-21.

[6] J. Cleary and L. Trigg, $\mathrm{K}^{*}$ : an instance-based learner using an entropic distance measure, in: Proceedings of the 12th International Conference on Machine Learning, 1995, pp. 108-114.

[7] W. Cohen, Fast effective rule induction, in: Machine Learning: Proceedings of the Twelfth International Conference, 1995.

[8] G. Demiroz and H. Guvenir, Classificatio by voting feature intervals, in: Proceedings of the 9th European Conference on Machine Learning, 1997, pp. 85-92.

[9] T. Dietterich, Machine-learning research:four current directions, AI Mag 18(4) (1997), 97-136.

[10] T. Dietterich, Ensemble methods in machine learning, in: Multiple Classifie s Systems: fi st international workshop; proceedings/MCS 2000, J. Kittler and F. Roli, eds, volume 1857 of Lecture Notes in Computer Science, Cagliari, Italy, June 2000. Springer, pp. 1-15.

[11] T. Dietterich and G. Bakiri, Solving multiclass learning problems via error-correcting output codesk, J Artif Intell Res 2 (1995), 263-286.

[12] R. Duda and P. Hart, Pattern Classificatio and Scene Analysis, Addison-Wesley, 1973.

[13] S. Dzeroski and B. Zenko, Stacking with multi-response model trees, in: Proceedings of Multiple Classifie Systems, Third International Workshop, MCS 2002, J.K. Fabio Roli, ed., Lecture Notes in Computer Science, Cagliari, Italy, 2002 Springer.

[14] S. Dzeroski and B. Zenko, Is combining classifier better than selecting the best one? Mach Learn 54(3) (2004), 255-273

[15] T. English, Stacked generalization and simulated evolution, Biosystems 39(16) (1996), 3-18.

[16] M. Faupel, http://www.micropraxis.com/gajit/index html, 1998.

[17] E. Frank and I. Witten, Generating accurate rule sets without global optimization, in: Proceedings of the Fifteenth International Conference on Machine Learning, Morgan Kaufmann, 1998, pp. 144-151.

[18] Y. Freund and R. Schapire, A decision-theoretic generalization of on-line learning and an application to boosting, in: Proceedings of the Second European Conference on Computational Learning Theory Springer-Verlag, ed., 1995, pp. 23-37.

[19] D. Goldberg, Genetic Algorithms in search, optimization, and machine learning, Addison-Wesley, 1989.

[20] L. Hansen and P. Salamon, Neural network emsembles, IEEE Trans Pattern Anal Mach Intell 12(10) (1990), 993-1001.

[21] J. Holland, Adaptation in Natural and Artificia Systems, The University of Michigan Press, 1975. 
[22] J. Holland, Adaptation in Natural and Artificia Systems, MIT Press, $2^{a}$ edition, 1992.

[23] W. Iba and P. Langley, Induction of one-level decision trees, in: Proceedings of the Ninth International Conference on Machine Learning, Morgan Kaufmann, 1992, pp. 233-240.

[24] G. John and P. Langley, Estimating continuous distribution in bayesian classifiers in: Proceedings of the Eleventh Conference on Uncertainty in Artificia Intelligence, M. Kaufmann, ed., 1995, pp. 338-345.

[25] R. Kohavi, The power of decision tables, in: Proceedings of the Eighth European Conference on Machine Learning, 1995.

[26] J. Kolen and J. Pollack, Back propagation is sensitive to initial conditions, in: Advances in Neural Information Processing Systems, 1991, pp. 860-867.

[27] A. Ledezma, R. Aler and D. Borrajo, Data Mining: a Heuristic Approach, chapter Heuristic Search Based Stacking of Classifiers Idea Group Publishing, 2001.

[28] B. Martin, Instance-based learning: Nearest neighbor with generalization. Master's thesis, University of Waikato, 1995.

[29] C. Merz, Using correspondence analysis to combine classifiers Mach Learn 36(1-2) (1999), 33-58.

[30] T. Mitchell, An Introduction to Genetic Algorithms, MIT Press, 1996.

[31] J. Quinlan, C4.5: Programs for Machine Learning. Morgan Kaufmann, San Mateo, CA, 1993.

[32] A. Seewald, How to make stacking better and faster while also taking care of an unknown weakness, in: Proceedings of the Nineteenth International Conference on Machine Learning (ICML 2002), A.G.H. Claude Sammut, ed., Sidney, Australia, July 2002. Morgan Kaufmann.

[33] A. Seewald and J. Fürnkranz, An evaluation of grading classifiers in: Advances in Intelligent Data Analysis, 4th International Conference, IDA 2001, Proceedings, F. Hoffmann, D.J. Hand, N.M. Adams, D.H. Fisher and G. Guimarães, eds, Lecture Notes in Computer Science, 2001, pp. 115-124.

[34] K. Ting and I. Witten, Issues in stacked generalization, J Artif Intell Res 10 (1999), 271-289.

[35] K.M. Ting and I. Witten, Stacked generalization: when does it work? in: Proceedings of the International Joint Conference on Artificia Intelligence, 1997.

[36] L. Todorovski and S. Dzeroski, Combining multiple models with meta decision trees, in: Proceedings of the 4th European Conference on Principles of Data Mining and Knowledge Discovery, 2000, pp. 54-64.

[37] I. Witten and E. Frank, Data mining: practical machine learning tools and techniques with Java implementations, Morgan Kaufmann, 2000.

[38] D. Wolpert, A mathematical theory of generalization: Part ii, Complex Syst 4 (1990), 201-249.

[39] D. Wolpert, Stacked generalization, Neural Netw 5 (1990), 241-259.

[40] Z. Zhou, J. Wu and W. Tang, Ensembling neural networks: Many could be better than al, Artif Intell 137(1-2), 2002. 